\title{
Toward Operational Compensation of Ionospheric Effects in SAR Interferograms: The Split-Spectrum Method
}

\author{
Giorgio Gomba, Alessandro Parizzi, Francesco De Zan, Michael Eineder, Senior Member, IEEE, and \\ Richard Bamler, Fellow, IEEE
}

\begin{abstract}
The differential ionospheric path delay is a major error source in L-band interferograms. It is superimposed to topography and ground deformation signals, hindering the measurement of geophysical processes. In this paper, we proceed toward the realization of an operational processor to compensate the ionospheric effects in interferograms. The processor should be robust and accurate to meet the scientific requirements for the measurement of geophysical processes, and it should be applicable on a global scale. An implementation of the split-spectrum method, which will be one element of the processor, is presented in detail, and its performance is analyzed. The method is based on the dispersive nature of the ionosphere and separates the ionospheric component of the interferometric phase from the nondispersive component related to topography, ground motion, and tropospheric path delay. We tested the method using various Advanced Land Observing Satellite Phased-Array type L-band synthetic aperture radar interferometric pairs with different characteristics: high to low coherence, moving and nonmoving terrains, with and without topography, and different ionosphere states. Ionospheric errors of almost $1 \mathrm{~m}$ have been corrected to a centimeter or a millimeter level. The results show how the method is able to systematically compensate the ionospheric phase in interferograms, with the expected accuracy, and can therefore be a valid element of the operational processor.
\end{abstract}

Index Terms-Interferometric synthetic aperture radar(InSAR), ionosphere estimation, split spectrum, synthetic aperture radar (SAR) ionospheric effects.

\section{INTRODUCTION}

$\mathbf{I}$ ONOSPHERIC propagation delay is one of the most relevant error sources in low-frequency spaceborne synthetic aperture radar (SAR) interferograms. SAR interferometry is a successful technique used to measure the Earth's topography and to study geophysical processes such as earthquakes,

Manuscript received May 26, 2015; revised August 25, 2015; accepted September 17, 2015.

G. Gomba, A. Parizzi, and F. De Zan are with the Remote Sensing Technology Institute, German Aerospace Center (DLR), 82234 Wessling, Germany (e-mail: giorgio.gomba@dlr.de).

M. Eineder is with the Remote Sensing Technology Institute, German Aerospace Center (DLR), 82234 Wessling, Germany, and also with Technische Universität München, 80333 Munich, Germany.

R. Bamler is with the Remote Sensing Technology Institute, German Aerospace Center (DLR), 82234 Wessling, Germany, and also with Lehrstuhl für Methodik der Fernerkundung, Technische Universität München, 80333 Munich, Germany.

Digital Object Identifier 10.1109/TGRS.2015.2481079 volcanoes, landslides, and glacier movements. Unfortunately, the accuracy of these measurements is limited by distortions that the ionosphere causes in the propagation of microwaves. In SAR interferograms, topography and ground deformation signals are mixed with ionospheric disturbances [1]. In order to avoid that the ionospheric propagation delay is confused with ground signals, it has to be estimated and removed [2]. The ionospheric distortions on the propagation of microwaves cause an additional group delay and a phase advance on SAR images, whose magnitude is inversely proportional to the frequency of the system. For this reason, L-band SAR systems such as the Advanced Land Observing Satellite (ALOS) Phased-Array type L-band SAR (PALSAR), its follow-up, i.e., ALOS-2, or the future Tandem-L, due to the lower frequency, experience more severe ionospheric distortions compared with C-band or $\mathrm{X}$-band systems.

The magnitude of ionospheric effects depends on the slant total electron content (TEC), which is the total number of electrons integrated between the satellite and the target, along a tube of $1 \mathrm{~m}^{2}$ cross section. If the correct TEC at the time of the two acquisitions is known, the TEC difference can be converted to a phase and removed from the interferogram. Global Navigation Satellite Systems (GNSS) dual-frequency systems can be used to estimate the TEC between the GNSS satellite and the ground and produce global or local vertical TEC maps [3]. These measures are unfortunately not detailed enough to be directly used to correct the ionospheric delay in L-band SAR interferograms [2].

Several methods to estimate the ionospheric differential TEC from SAR data, with higher precision compared with the GNSS-based measurements, have been proposed in the past years [4] and are briefly summarized in the following. The range split-spectrum method exploits the dispersive propagation of the ionosphere to separate the ionospheric-related phase term from the nondispersive phase term of an interferogram [5], [6]. The range phase-group delay difference method estimates the ionosphere, taking advantage of the fact that the ionospheric phase and group delays have opposite signs, unlike groundrelated phase and group delays, which have the same sign [5], [7]. The azimuth shift method exploits the proportional relation between differential azimuth shift and the azimuth derivative of the differential ionosphere [7]-[9]. Multiple aperture interferometry is sometimes used to estimate the azimuth shifts and can be considered an equivalent method [10]. These two methods cannot recover the ionospheric range variations, being sensitive 
just along the azimuth direction. The Faraday rotation method requires quad-polarized measurements to estimate the Faraday rotation angle and TEC from the individual images [11]-[13]. By differencing the derived TEC information, differential TEC maps can be produced and converted to ionospheric phase screens. However, the Faraday rotation depends on the geometry between the radar acquisition and the Earth magnetic field. For this reason, the method's sensitivity is strongly reduced in an area of several hundred kilometers along the magnetic equator. Therefore, in addition to requiring full-pol data sets, this approach has the disadvantage of not being globally applicable.

These techniques have been proven to work, but an evaluation of their performances and suitability for an operational environment on a global scale is still missing. In this paper, we demonstrate how the range split-spectrum method is capable of estimating the differential ionospheric path delay, increasing the performance of interferometric SAR (InSAR) techniques and, hence, improving the measurement accuracy of geophysical processes. To improve the method's robustness, its weaknesses are analyzed together with possible sources of systematic biases; solutions to mitigate these problems are proposed. Finally, to show its wide applicability, the improved method has been tested with a variety of different L-band ALOS PALSAR images. The data include different coherence levels, different environmental conditions such as nonmoving and moving terrains, with and without topography, and different ionospheric conditions. Images with particular ionosphere phenomena such as aurora borealis are also used. The results show that the method is effectively able to compensate the ionospheric effects in interferograms, that the obtained accuracy is comparable with the expected one, and that the method can be easily applied to new test cases almost without tuning and can therefore be included in an operational processor.

In Section II of this paper, the ionospheric effects on the propagation of microwaves are summarized. Their estimation using the range split-spectrum method is presented along with its accuracy. In Section III, an overall scheme of the implementation of the method is presented, and its critical points are analyzed in detail. The method is applied to L-band ALOS PALSAR images; Section IV presents the tests results. In Section $\mathrm{V}$, the findings are summarized, and future work is proposed.

\section{THEORETICAL BACKGROUND}

\section{A. Ionospheric Effects on Interferograms}

The ionosphere is the portion of the Earth's upper atmosphere where ions and electrons are present with sufficient density to significantly affect the propagation of radio waves. Charged particles are created by the incoming solar radiation that ionizes atmospheric gases. Their concentration in the ionosphere varies with the altitude but normally has a peak between 300 and $400 \mathrm{~km}$. The 3-D structure of the ionosphere is often approximated by an idealized thin layer, which is positioned at the barycenter of the electron density. Two effects of the ionosphere on a traversing microwave can be derived from the Appelton-Hartree equation [14], which relates the refractive index of the plasma to its ionization. The first effect is

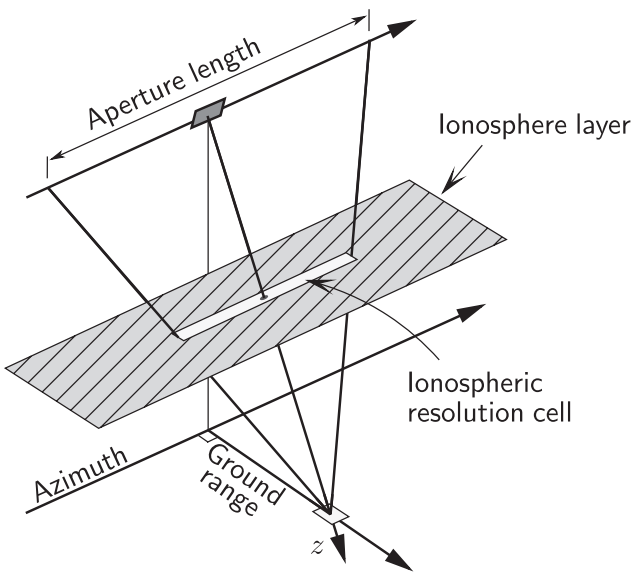

Fig. 1. Geometry of the system. The ionospheric resolution is limited by the SAR range resolution and by the synthetic aperture length.

a phase advance of the carrier. This is calculated by integrating the density of free electrons $n_{e}$ along the two-way wave path, i.e.,

$$
\phi_{\text {iono }}(f)=2 \cdot \frac{2 \pi K}{c f} \int n_{e}(z) d z=\frac{4 \pi K}{c f} \mathrm{TEC} .
$$

In the latter, $f$ is the carrier frequency, $c$ is the speed of light in vacuum, and $K=40.28 \mathrm{~m}^{3} / \mathrm{s}^{2}$. The slant TEC $=\int n_{e}(z) d z$ is the TEC experienced by the radio wave; it can be converted to a vertical TEC using a mapping function [15].

As the ionosphere is dispersive, different frequencies are differently advanced according to (1). The second effect is a rotation of the polarization angle, a phenomenon known as Faraday rotation. Since its contribution to the interferometric phase is minimal, it will be neglected in the following.

The interferometric phase is the sum of different components, i.e.,

$\Delta \phi=\frac{4 \pi f_{0}}{c}\left(\Delta r_{\text {topo }}+\Delta r_{\text {mov }}+\Delta r_{\text {tropo }}\right)-\frac{4 \pi K}{c f_{0}} \Delta \mathrm{TEC}$

where $f_{0}$ is the carrier frequency; and $\Delta r_{\text {topo }}, \Delta r_{\text {mov }}$, and $\Delta r_{\text {tropo }}$ are the topographic path delay, which includes the flat-earth phase and the topography-related phase, the differential path delay associated with a ground movement between acquisitions, and the differential tropospheric path delay, respectively. We group the nondispersive contributions to the interferometric phase in

$$
\Delta \phi_{\text {non-disp }}=\frac{4 \pi f_{0}}{c}\left(\Delta r_{\text {topo }}+\Delta r_{\text {mov }}+\Delta r_{\text {tropo }}\right)
$$

to distinguish them from the dispersive ionospheric contribution

$$
\Delta \phi_{\text {iono }}=-\frac{4 \pi K}{c f_{0}} \Delta \mathrm{TEC}
$$

where $\triangle$ TEC is the differential TEC, i.e., the TEC difference between the two acquisitions. The negative sign of (2) and (4) indicates that the ionospheric contribution is a phase advance.

The ionospheric azimuth resolution is limited by the synthetic aperture length projected at the height of the ionosphere layer, as illustrated in Fig. 1. The ionospheric phase screen, 
observed in the interferogram, is therefore a low-pass version of the real, possibly turbulent, ionosphere. Variations of the ionospheric path delay within the ionospheric azimuth resolution produce a nonnominal phase history which causes azimuth effects such as shift and blurring. These effects reduce the interferometric coherence. The magnitude and correlation length of the variations, with respect to the azimuth resolution in the ionosphere, determine the type and intensity of the effects. A linear trend of the ionospheric TEC along the flight path of the satellite causes an azimuth shift, whereas any deviation from a linear trend defocuses the image. Azimuth shifts are corrected by the coregistration and resampling steps, whereas a correction of the defocusing would require additional processing. Subapertures or a semifocusing processing can be used to increase the resolution [16], [17] and improve those situations where the azimuth variations are so strong that they cause defocusing and loss of interferometric coherence. For all other cases, the coregistration and resampling are normally enough to recover the coherence. To produce an ionosphere-free interferogram, it is sufficient to estimate and remove the low-pass ionosphere which is superimposed to the interferogram (the ionospheric phase). In this sense, if the ionospheric variations, with respect to the aperture length, are moderate enough that they do not cause relevant azimuth defocusing or coherence losses after coregistration, the ionosphere can be considered smooth, and there is no need to increase the ionospheric resolution using subapertures or semifocusing.

The dispersive ionospheric contribution $\Delta \phi_{\text {iono }}$ is inversely proportional to the frequency, whereas the nondispersive ground and troposphere contribution $\Delta \phi_{\text {non-disp }}$ is directly proportional to the frequency. This characteristic can be exploited to separate these two phase components: to establish the maximum possible separation accuracy, we calculate the Cramér-Rao bound (CRB).

\section{B. CRB for Ionospheric Path Delay Estimation}

We use the CRB to calculate the maximum achievable accuracy of estimating the ionospheric path delay, considering the information [which originates from the effect in (1)] that can be obtained from range signals. To derive the CRB estimate, we assume that the SAR acquisitions are dominated by distributed scatterers, such that the observed SAR signals can be represented by partially correlated complex Gaussian signals. The acquisitions can be modeled without loss of generality in the frequency domain, i.e.,

$$
\begin{aligned}
S_{1}(f)= & \sqrt{\gamma} A(f)+\sqrt{1-\gamma} W_{1}(f) \\
S_{2}(f)= & \sqrt{\gamma} A(f) \exp \left(-j \Delta \phi_{\text {non-disp }} \frac{f}{f_{0}}-j \Delta \phi_{\text {iono }} \frac{f_{0}}{f}\right) \\
& +\sqrt{1-\gamma} W_{2}(f)
\end{aligned}
$$

where $A(f), W_{1}(f)$, and $W_{2}(f)$ are uncorrelated complex Gaussian signals, with zero mean, unitary variance, and a white spectrum of bandwidth $B$ and central frequency $f_{0}$. $A$ represents the coherent scattering, whereas $W_{1}$ and $W_{2}$ represent the decorrelation noise; $\gamma$ is the magnitude of the interferometric coherence. The interferometric phase is assigned to the second acquisition.

For each frequency, the two observations are collected in the vector $\boldsymbol{y}(f)=\left[S_{1}(f), S_{2}(f)\right]$. The elements of the Fisher information matrix, calculated using the covariance $C(f)=$ $E\left[\boldsymbol{y}(f) \boldsymbol{y}^{H}(f)\right]$, are given by [18]

$$
[\mathbf{F I M}(f)]_{n, k}=\operatorname{tr}\left\{\boldsymbol{C}^{-1}(f) \frac{\delta \boldsymbol{C}(f)}{\delta \theta_{n}} \boldsymbol{C}^{-1}(f) \frac{\delta \boldsymbol{C}(f)}{\delta \theta_{k}}\right\}
$$

where $n, k=\{1,2\}$; and $\theta_{1}$ and $\theta_{2}$ are the two unknown parameters $\Delta \phi_{\text {non-disp }}$ and $\Delta \phi_{\text {iono }}$, respectively. The result is

$$
\operatorname{FIM}(f)=\frac{2 \gamma^{2}}{1-\gamma^{2}}\left[\begin{array}{cc}
\frac{f^{2}}{f_{0}^{2}} & 1 \\
1 & \frac{f_{0}^{2}}{f^{2}}
\end{array}\right] .
$$

The final Fisher information matrix can be obtained by integrating across the signal spectrum and multiplying by the number of independent samples $N$ [19], [20], i.e.,

$$
\mathbf{F I M}=\frac{N}{B} \int_{f_{0}-B / 2}^{f_{0}+B / 2} \mathbf{F I M}(f) d f .
$$

The CRB for the two parameters $\Delta \phi_{\text {non-disp }}$ and $\Delta \phi_{\text {iono }}$ is the inverse of the Fisher information matrix, i.e.,

$$
\mathrm{CRB}=\frac{f_{0}^{2}}{B^{2}} \frac{3}{2 N} \frac{1-\gamma^{2}}{\gamma^{2}}\left[\begin{array}{cc}
1 & \frac{B^{2}}{4 f_{0}^{2}}-1 \\
\frac{B^{2}}{4 f_{0}^{2}}-1 & \left(1-\frac{B^{2}}{4 f_{0}^{2}}\right)^{2}\left(1+\frac{B^{2}}{12 f_{0}^{2}}\right)
\end{array}\right] .
$$

From the latter, we obtain the standard deviation of the ionospheric phase estimate

$$
\sigma_{\Delta \mathrm{TEC}} \geq \frac{c f_{0}}{4 \pi K} \cdot[\mathrm{CRB}]_{2,2} \approx \frac{c f_{0}^{2}}{4 \pi \mathrm{KB}} \sqrt{\frac{3}{2 N}} \frac{\sqrt{1-\gamma^{2}}}{\gamma} .
$$

Expressing this accuracy in meters, we obtain the precision with which the error component of the ground motion estimation, related to the ionospheric noise, can be estimated, i.e.,

$$
\sigma_{\Delta r_{\mathrm{mov}}} \geq \frac{c}{4 \pi f_{0}} \cdot[\mathrm{CRB}]_{1,1}=\frac{c}{4 \pi B} \sqrt{\frac{3}{2 N}} \frac{\sqrt{1-\gamma^{2}}}{\gamma}
$$

It is interesting to note that the latter is equal to the group delay estimation accuracy [21], divided by two. The additional 0.5 factor is due to the fact that the ionosphere contributes both to the phase and group delays, but with opposite signs. The precision limit, in estimating the ionosphere, is then set by the estimation accuracy of the group delay.

The estimated ionospheric phase and its accuracy are relative to the resolution cell in the ionosphere. This is limited in range by the SAR image resolution and by the multilooking factor and in azimuth by the aperture length projected at the height of the ionosphere. Ionospheric variations with a finer spatial scale than the resolution are neither measured by the interferogram nor can they be estimated (unless using other techniques, such as subapertures or semifocusing). 


\section{Range Split-Spectrum Method}

The range split-spectrum method [5], [6] suggests to exploit the different frequency behavior of the two components of (2) to separate them. The procedure consists in the generation of two range subbands (indices $L$ for the lower subband and $H$ for the higher subband) with center frequencies $f_{L}$ and $f_{H}$. The interferograms computed from each subband yield the phases $\Delta \phi_{L}$ and $\Delta \phi_{H}$. Rewriting (2) for the two interferograms in terms of nondispersive and dispersive effects, we have

$$
\begin{aligned}
\Delta \phi_{L} & =\Delta \phi_{\text {non-disp }} \frac{f_{L}}{f_{0}}+\Delta \phi_{\text {iono }} \frac{f_{0}}{f_{L}} \\
\Delta \phi_{H} & =\Delta \phi_{\text {non-disp }} \frac{f_{H}}{f_{0}}+\Delta \phi_{\text {iono }} \frac{f_{0}}{f_{H}} .
\end{aligned}
$$

Inverting these equations, the dispersive $\Delta \phi_{\text {iono }}$ and nondispersive $\Delta \phi_{\text {non-disp }}$ components of the delay can be estimated, i.e.,

$$
\begin{aligned}
\Delta \hat{\phi}_{\text {iono }} & =\frac{f_{L} f_{H}}{f_{0}\left(f_{H}^{2}-f_{L}^{2}\right)}\left(\Delta \phi_{L} f_{H}-\Delta \phi_{H} f_{L}\right) \\
\Delta \hat{\phi}_{\text {non-disp }} & =\frac{f_{0}}{\left(f_{H}^{2}-f_{L}^{2}\right)}\left(\Delta \phi_{H} f_{H}-\Delta \phi_{L} f_{L}\right) .
\end{aligned}
$$

This simple mathematical operation requires some care in the practical implementation. The method can in fact be realized in different ways, possibly reaching the correct estimation of the ionospheric phase. However, there are some critical steps which could lead to a poor result if not carefully implemented. The interferometric coregistration, for instance, should be able to estimate strong ionospheric azimuth shifts, in order to correct them and recover the coherence. Another issue arises from phase unwrapping: given that the lower and upper interferograms have to be unwrapped prior to the scaling, eventual errors lead to a bias in the estimation. Finally, the interferometric phase noise, which is strongly amplified by the upscaling, has to be reduced. The resulting estimation accuracy will depend on the bandwidth, coherence, multilooking, and noise filtering. In Section III, we propose an implementation, and we focus on some critical steps which were carefully analyzed to improve the final result.

\section{Split-Spectrum Method Accuracy}

In [5], it is shown that the accuracy of the ionospheric phase estimate is maximized when the bandwidth of each subband is one third of the total bandwidth. For high coherence and large $N$, the accuracy is approximated using the interferometric phase variance of the subbands [22], i.e.,

$$
\sigma_{\Delta \phi_{H, L}}^{2}=\frac{1}{2 N_{s b}} \frac{1-\gamma^{2}}{\gamma^{2}}=\frac{3}{2 N} \frac{1-\gamma^{2}}{\gamma^{2}}
$$

where $\gamma$ is the interferometric coherence. The number of independent samples used in each interferogram $N_{s b}$ is one third of the total $N$, since only one third of the bandwidth is used. Given that the two interferograms are uncorrelated, from (13) we can write

$$
\sigma_{\Delta \hat{\phi}_{\text {iono }}}^{2}=\left(\frac{f_{L} f_{H}}{f_{0}\left(f_{H}^{2}-f_{L}^{2}\right)}\right)^{2}\left(f_{H}^{2} \sigma_{\Delta \phi_{L}}^{2}+f_{L}^{2} \sigma_{\Delta \phi_{H}}^{2}\right) .
$$

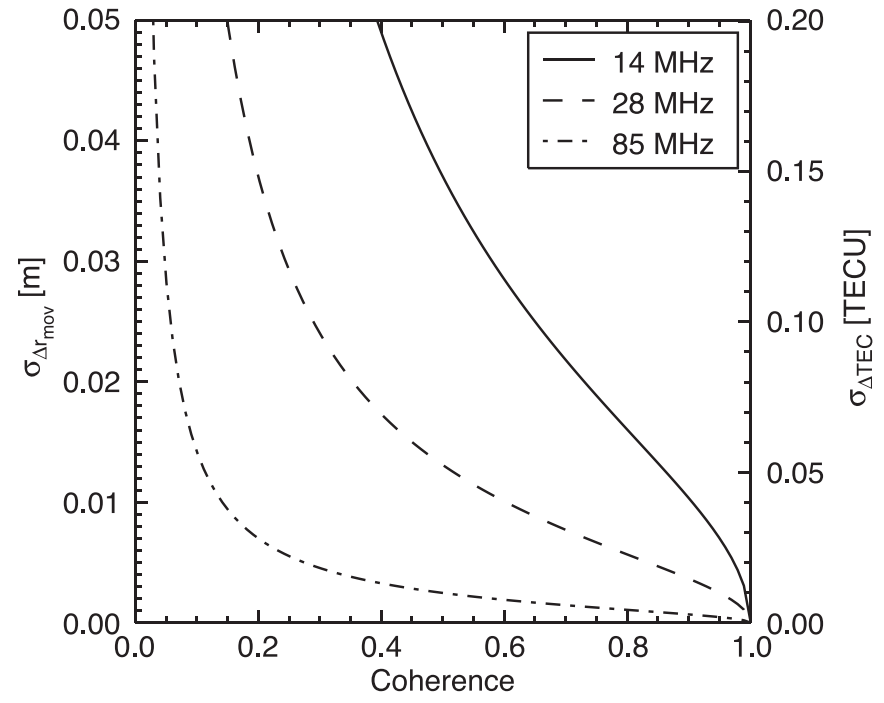

Fig. 2. Standard deviation of the ground movement (left axis) and ionospheric phase (right axis) estimation, for a ground area of $1 \mathrm{~km}^{2}$, as a function of the interferometric coherence. Different range bandwidths of (solid line) $14 \mathrm{MHz}$, (dashed line) $28 \mathrm{MHz}$, and (dash-dot line) $85 \mathrm{MHz}$ are used. Carrier frequency is $1.27 \mathrm{GHz}$.

Supposing that the coherences of both interferograms are equal, the ionospheric phase accuracy, from (14) and (15), is

$$
\begin{aligned}
\sigma_{\Delta \hat{\phi}_{\mathrm{iono}}} & =\left(\frac{f_{L} f_{H}}{f_{0}\left(f_{H}^{2}-f_{L}^{2}\right)}\right) \sqrt{f_{L}^{2}+f_{H}^{2}} \cdot \sigma_{\Delta \phi_{H, L}} \\
& \approx \frac{3 f_{0}}{4 B} \sqrt{\frac{3}{N}} \frac{\sqrt{1-\gamma^{2}}}{\gamma}
\end{aligned}
$$

which, when converted to TECs, becomes

$$
\sigma_{\Delta \mathrm{TEC}}=\frac{3 c f_{0}^{2}}{16 \pi \mathrm{KB}} \sqrt{\frac{3}{N}} \frac{\sqrt{1-\gamma^{2}}}{\gamma} .
$$

In reality, the coherences of the interferograms can differ; the exact accuracy is then just a bit more complex than (16). It includes both coherences, as well as the range and azimuth oversampling factors. To simplify the discussion, in the following, we will use the shorter (16). As it can be seen, comparing (17) and (10), the split-band method accuracy is only 1.06 times worse than the CRB. The estimation accuracy converted in meters is

$$
\sigma_{\Delta r_{\mathrm{mov}}}=\frac{3 c}{16 \pi B} \sqrt{\frac{3}{N}} \frac{\sqrt{1-\gamma^{2}}}{\gamma} .
$$

Equations (17) and (18) are represented in Fig. 2. The number of independent samples is calculated for a ground area size of $1 \mathrm{~km}^{2} ; 1.27-\mathrm{GHz}$ carrier frequency; $14-, 28-$, and $85-\mathrm{MHz}$ range bandwidths; 5-m azimuth resolution; and $30^{\circ}$ incidence angle. For example, a coherence of 0.6 allows an accuracy of about $1 \mathrm{~cm}$ when performing the multilooking on an area of $1 \mathrm{~km}^{2}$ using images with $28-\mathrm{MHz}$ bandwidth. One centimeter seems already an acceptable accuracy because it is comparable with a typical residual tropospheric influence after compensation. However, the accuracy can be increased by further filtering: a discussion about multilooking and filtering can be found in Section III. 


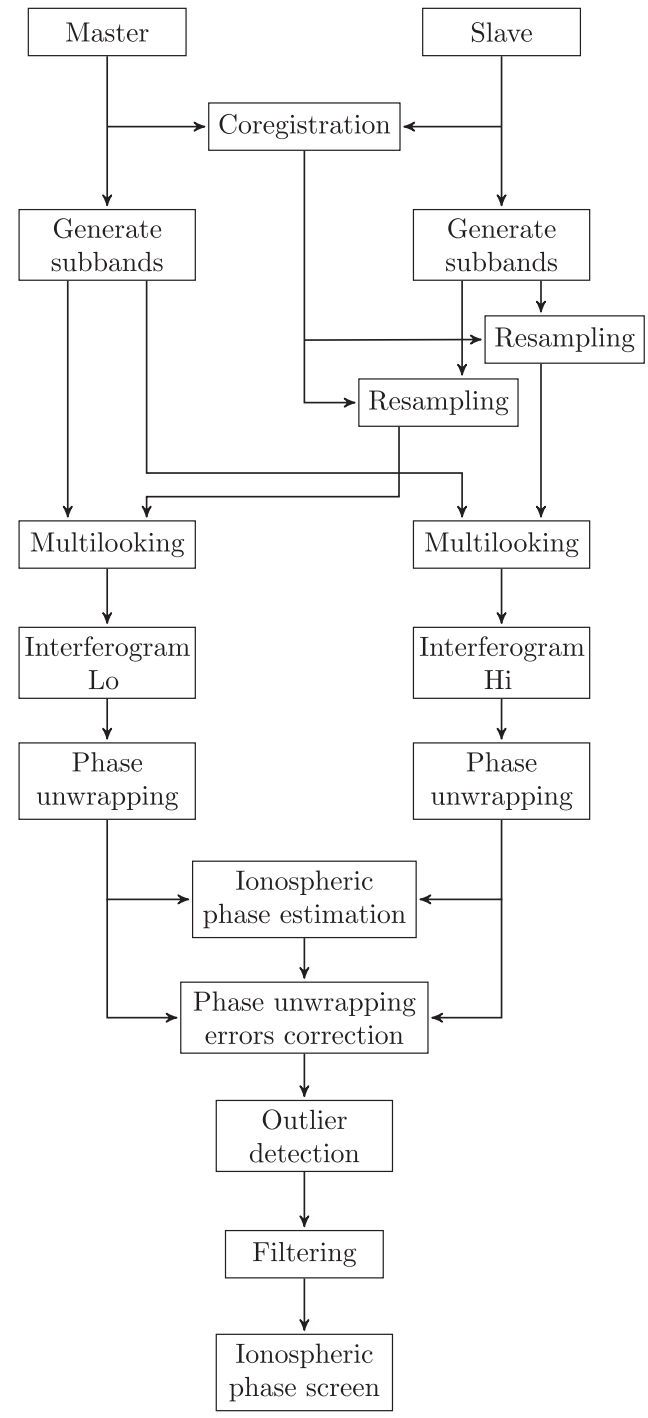

Fig. 3. Implementation of the split-spectrum method.

\section{IMPLEMENTATION AND SYSTEMATIC ERRORS}

Here follows a description of the proposed implementation of the split-spectrum method. In Fig. 3, a schematic representation is reported.

First, the images have to be carefully coregistered; to improve robustness, in our implementation, we use a mixture of coherent patch-based cross correlation for high-coherence areas and incoherent cross correlation for low-coherence areas. In order to ensure that, in case of strong ionospheric azimuth variations and/or ground movements, the high-frequency components of the motion field are preserved, no polynomial fitting of the shifts is performed.

Considering the wavenumber shift [23], in the second step, common range band filtering is performed; this increases the coherence for pairs with nonzero normal baseline. In Section III-E, wavenumber-shift-related aspects are further discussed. Two subbands of one third of the total common bandwidth are then generated by bandpass filtering. The slave images are resampled using the shifts which have been estimated during the coregistration step. Azimuth shifts generated by ionospheric variations are thus corrected and the coherence recovered.

After resampling, an interferogram is calculated from each subband; orbit information and a digital elevation model (DEM) are used to compensate the topographic phase. The amount of multilooking is discussed in Section III-A. Interferograms still contain the differential phase due to ground movements between acquisitions, the atmospheric phase, and the ionospheric phase. Both interferograms are unwrapped using a minimum cost flow algorithm. The effect of possible phase unwrapping errors is discussed in Section III-C. The dispersive and nondispersive components are separated by using (13). Differential phase unwrapping errors are then corrected as presented in Section III-C.

An outlier detection step is necessary to eliminate those pixels that do not follow a Gaussian distribution. This is performed using a robust median moving filter and the theoretical standard deviation (16), calculated using the interferograms coherences.

The ionospheric phase estimates are then filtered, to reach, if possible, the desired accuracy. The filtering step is described in Section III-B. Finally, the ionospheric phase screen is removed from the full-band interferogram obtaining an ionospherecompensated interferogram.

\section{A. Multilooking}

In this implementation, there are two filtering (averaging) steps: the first one is the multilooking performed during the interferogram generation on the complex data, and the second one is the filtering of the estimated ionospheric phase. The amount of multilooking and final filtering can be partly interchanged. However, a minimum initial multilooking has to be done to reach the efficient and asymptotic estimation of the phase [24]. Moreover, it has, as usual, to be realized such that the number of looks is small enough to obtain high resolution and no coherence losses due to rapid fringes but also big enough such that phase unwrapping is possible. The multilooking factor is then constrained by these requisites. Thereafter, one can decide how to perform the final filtering.

\section{B. Filtering}

Since the ionosphere is usually relatively smooth, the estimates are often spatially correlated. This suggests that a filtering step, which removes the high-frequency noise components, could help to increase the accuracy. It is then more convenient to filter the ionosphere estimate rather than the ground component estimate, because the former is usually spatially smoother than the latter. The filtered phase screen is then subtracted from the full-band interferogram to obtain an ionosphere-compensated interferogram. The final precision is related to the phase screen accuracy. The amount of filtering depends on the desired final precision with respect to the variance of the initial estimate and needs to be decided after some careful considerations.

In our implementation, a 2-D Gaussian weighted filter is used to smooth the ionospheric phase. The filter is the normalized product of two identical 1-D Gaussian functions with variance $M^{2} / 4 \pi$ in range and azimuth. It reduces the phase variance 
by a factor equal to the effective number of looks, which is approximately $M^{2}$. The precise effective number of looks $N_{\text {eff }}$ can be obtained by numerical integration. The parameter $M$, which is needed to reach a desired accuracy $\sigma_{\Delta r_{\text {mov }}}$, can be calculated with respect to the initial accuracy. From (18), we obtain the relationship

$$
M=\frac{3 c}{16 \pi B} \sqrt{\frac{3}{N}} \frac{\sqrt{1-\gamma^{2}}}{\gamma} \frac{1}{\sigma_{\Delta r_{\mathrm{mov}}}^{2}}
$$

where $N$ is, as before, the multilooking factor.

Apart from the Gaussian window, the optimum approach to filter the ionosphere estimates is based on the maximumlikelihood principle [18]. We use a weighted mean, where the weights are the inverse of the expected variance, calculated from the interferograms coherence. Outliers, which have been detected in the previous step, are not used by giving them a weight equal to zero. The coefficients of the weighted mean, which are used to calculate the filtered ionosphere $\overline{\Delta \phi_{\text {iono }}}(i, k)$, combine the Gaussian filter $g$ (having its peak at $\{i, k\}$ ) and the expected variance

$$
w(i, k)=K \frac{g(i, k)}{\sigma_{\Delta \hat{\phi}_{\text {iono }}}^{2}(i, k)}
$$

where $K$ is a normalization factor such that $\sum w(i, k)=1$. The variance of the result can be calculated as

$$
\begin{aligned}
\sigma_{\frac{\Delta \phi_{\text {iono }}}{2}}(i, k) & =\sum w_{i}^{2}(i, k) \sigma_{\Delta \hat{\phi}_{\text {iono }}}^{2}(i, k) \\
& =K^{2} \sum \frac{g^{2}(i, k)}{\sigma_{\Delta \hat{\phi}_{\text {iono }}}^{2}(i, k)}
\end{aligned}
$$

In practice, the filtering can be realized with convolutions (indicated by $*$ ), i.e.,

$$
\begin{aligned}
\overline{\Delta \phi_{\text {iono }}} & =\frac{\Delta \phi_{\text {iono }} / \sigma_{\Delta \hat{\phi}_{\text {iono }}^{2}}^{2} * g}{1 / \sigma_{\Delta \hat{\phi}_{\text {iono }}}^{2} * g} \\
\sigma_{\bar{\Delta} \phi_{\text {iono }}}^{2} & =\frac{1 / \sigma_{\Delta \hat{\phi}_{\text {iono }}}^{2} * g^{2}}{\left(1 / \sigma_{\Delta \hat{\phi}_{\text {iono }}}^{2} * g\right)^{2}} .
\end{aligned}
$$

Ionospheric variations with a spatial scale smaller than the smoothing window are smoothed out and not recovered. Then a tradeoff exists between reducing the estimation noise and reducing the bias due to excessive smoothing, and it could be difficult to decide the size of the filter window, i.e., the parameter $M$. Anisotropic filters or adaptive filters could help to increase the accuracy without introducing biases. The combination of different ionosphere estimation methods is also a possibility that is being studied [4], [9].

\section{Phase Unwrapping Errors}

Here, we analyze the effect of phase unwrapping errors on the estimation of the ionospheric phase. In particular, if the coherence is low, phase unwrapping errors can occur and introduce biases in the ionosphere estimation. Phase unwrapping is performed separately on each subband, and phase unwrapping errors can therefore be different. Let us define phase unwrapping errors in the lower and upper subbands with $2 \pi m$ and $2 \pi(m+d)$, respectively. This way, $m$ is a common phase unwrapping error, and $d$ is a differential one. Both terms are integers and not necessarily constant within the interferogram. Equation (12) becomes

$$
\begin{aligned}
\Delta \phi_{L} & =\Delta \phi_{\text {non-disp }} \frac{f_{L}}{f_{0}}+\Delta \phi_{\text {iono }} \frac{f_{0}}{f_{L}}+2 \pi m \\
\Delta \phi_{H} & =\Delta \phi_{\text {non-disp }} \frac{f_{H}}{f_{0}}+\Delta \phi_{\text {iono }} \frac{f_{0}}{f_{H}}+2 \pi(m+d) .
\end{aligned}
$$

The estimated ionospheric phase thus yields

$$
\Delta \hat{\phi}_{\text {iono }} \approx \Delta \phi_{\text {iono }}+\pi m+\frac{\pi}{2} d-\frac{3 \pi f_{0}}{2 B} d .
$$

The latter shows the different behavior of common phase unwrapping errors and differential ones.

1) Differential Phase Unwrapping Error: The term $d$ is scaled by the factor $f_{0} / B$, generating a significant bias that should be removed. Taking the difference between the two interferograms, one has

$\Delta \phi_{L}-\Delta \phi_{H} \approx-\frac{2 B}{3 f_{0}} \Delta \phi_{\text {non-disp }}+\frac{2 B}{3 f_{0}} \Delta \phi_{\text {iono }}-2 \pi d$

whereas their sum yields

$\Delta \phi_{L}+\Delta \phi_{H} \approx 2 \Delta \phi_{\text {non-disp }}+2 \Delta \phi_{\text {iono }}+4 \pi c+2 \pi d$.

The term $d$ can be then estimated from the phase difference (26), i.e.,

$$
\hat{d}=\left\lfloor\frac{1}{2 \pi}\left(\Delta \phi_{H}-\Delta \phi_{L}-\frac{2 B}{3 f_{0}} \Delta \phi_{\text {non-disp }}+\frac{2 B}{3 f_{0}} \Delta \phi_{\text {iono }}\right)\right\rceil
$$

where $\lfloor\cdot 7$ indicates the rounding to the nearest integer. Even if $\Delta \phi_{\text {non-disp }}$ and $\Delta \phi_{\text {iono }}$ are not known with high accuracy, they do not lead to large biases in the measure of $d$ since they are reduced by the scaling term $2 B /\left(3 f_{0}\right)$. If needed, an iterative procedure can be implemented to reach the correct values.

An example of the results obtained by applying this correction is reported in Section IV-C2.

2) Common Phase Unwrapping Error: The recovery of the common term $m$ is more delicate; using the phase sum (27), one has

$$
\hat{m}=\left\lfloor\frac{1}{4 \pi}\left(\Delta \phi_{L}+\Delta \phi_{H}-2 \Delta \phi_{\text {non-disp }}-2 \Delta \phi_{\text {iono }}\right)-\frac{d}{2}\right\rceil .
$$

Inaccurate $\Delta \phi_{\text {non-disp }}$ and $\Delta \phi_{\text {iono }}$ are misinterpreted as phase unwrapping errors, leading to an even more inaccurate estimate of $\Delta \phi_{\text {non-disp }}$ and $\Delta \phi_{\text {iono. }}$ Larger filtering windows can improve the accuracy of $\Delta \phi_{\text {non-disp }}$ and $\Delta \phi_{\text {iono }}$ but also lower the resolution. This can be unacceptable, particularly for the ground-related phase, which is more likely to be spatially variable. The success of this method depends on the scene characteristics (high coherence or smooth signals) and is therefore not robust enough. A common phase unwrapping error, on the 
other hand, has a small impact, compared with a differential one. Moreover, it can be considered negligible if its magnitude is smaller than the accuracy (17), as it usually happens for small error areas.

Wide areas with ambiguous phase unwrapping, e.g., when a river cut an image in two parts, can neither be recovered nor simply ignored. This is a general problem for interferometry; radargrammetry or GPS measurements could be normally used to solve it. Unfortunately, the presence of the ionosphere makes the radargrammetry method inapplicable, as discussed in the next session.

\section{Unambiguous Ionospheric Phase}

The estimated differential ionospheric phase can be only known to within a constant offset, since both interferograms are not absolute phases. The split-spectrum estimate is then only a relative differential phase, not an absolute differential phase. Radargrammetry is also subject to the ionospheric influence; in fact, the group delay $\Delta \tau$ is increased by the presence of the ionosphere, i.e.,

$$
2 f_{0} \Delta \tau=\frac{4 \pi f_{0}}{c}\left(\Delta \tau_{\text {topo }}+\Delta \tau_{\text {mov }}+\Delta \tau_{\text {tropo }}\right)+\frac{4 \pi K}{c f_{0}} \Delta \text { TEC. }
$$

With respect to (2), the ionospheric term has, in the latter, the opposite sign. This property is used in the phase-group delay difference method [5], [7] to estimate the ionospheric contribution by subtracting the unwrapped phase from the radargrammetry. The difference contains both the ionosphere and the absolute phase offset, which cannot be separated.

It is possible to estimate the unambiguous phase by applying the same principle of the split-spectrum method to the radargrammetric shifts of the subbands. Unfortunately, the resulting accuracy is very low, i.e.,

$$
\sigma_{\Delta \hat{\phi}_{\text {iono }}} \approx \frac{9 f_{0}^{2}}{2 B^{2}} \sqrt{\frac{1}{N}} \frac{\sqrt{1-\gamma^{2}}}{\gamma}
$$

making this procedure unlikely applicable.

\section{E. Wavenumber Shift}

The wavenumber shift effect [23] has to be considered, while generating the subbands, to only take the common band and thus increase the coherence. However, since different parts of the ground reflectivity spectrum are linked to different signal frequencies, the matching of differently shifted ground spectra (between two images) aligns bands with different central frequencies. In [25], it is suggested that, due to this effect, each image has a different carrier frequency and therefore experiences a different ionospheric phase advance. Let us reformulate (4) as follows:

$$
\Delta \phi_{\text {iono }}=\frac{4 \pi K}{c}\left(\frac{\mathrm{TEC}_{1}}{f_{0}-\Delta f / 2}-\frac{\mathrm{TEC}_{2}}{f_{0}+\Delta f / 2}\right)
$$

where $\mathrm{TEC}_{1}$ and $\mathrm{TEC}_{2}$ are the TEC levels during the first and second acquisitions, respectively; and $\Delta f$ is the spectral shift [23]. Considering that $\Delta f \ll f_{0}$, the latter can be reduced to

$$
\Delta \phi_{\mathrm{iono}} \approx \frac{4 \pi K}{c f_{0}} \Delta \mathrm{TEC}+\frac{4 \pi K}{c f_{0}} \frac{\Delta f}{2 f_{0}} \Sigma \mathrm{TEC}
$$

where $\triangle \mathrm{TEC}$ is, as in (4), the differential TEC between acquisitions, and $\Sigma T E C=\mathrm{TEC}_{1}+\mathrm{TEC}_{2}$, i.e., the sum of the two TEC levels. We rewrite (12) to take into account also the last term of (33), i.e.,

$$
\begin{aligned}
\Delta \phi_{L} & =\Delta \phi_{\text {non-disp }} \frac{f_{L}}{f_{0}}+\Delta \phi_{\Delta \text { iono }} \frac{f_{0}}{f_{L}}+\Delta \phi_{\Sigma \text { iono }} \frac{f_{0}}{f_{L}} \frac{\Delta f}{2 f_{L}} \\
\Delta \phi_{H} & =\Delta \phi_{\text {non-disp }} \frac{f_{H}}{f_{0}}+\Delta \phi_{\Delta \text { iono }} \frac{f_{0}}{f_{H}}+\Delta \phi_{\text {¿iono }} \frac{f_{0}}{f_{H}} \frac{\Delta f}{2 f_{H}}
\end{aligned}
$$

where $\Delta \phi_{\Delta \text { iono }}=\left(4 \pi K / c f_{0}\right) \Delta \mathrm{TEC}$, and $\Delta \phi_{\Sigma i \text { iono }}=(4 \pi K /$ $\left.c f_{0}\right) \Sigma T E C$. The estimated ionospheric phase becomes thus

$$
\Delta \hat{\phi}_{\mathrm{iono}} \approx \Delta \phi_{\Delta \mathrm{iono}}+\frac{3 \Delta f}{4 f_{0}} \Delta \phi_{\text {¿iono }}
$$

this shows that the absolute ionosphere biases the differential ionosphere estimate. A compensation of the absolute ionosphere bias could be done using data from different sources, such as GNSS-based TEC maps. On the other hand, an estimation of the absolute ionosphere could be possible if the spectral shift varies within the same ionospheric level.

Relative variations of the terms of (35) generate biases in the estimated phase. To get an idea of the order of magnitude of the possible biases, we analyze two examples.

Consider an ionospheric spatial gradient in midlatitude regions; a long acquisition spanning some hundreds of kilometers could encounter a relative spatial change of $\Sigma T E C$ on the order of some tens of TECU, and, supposing similar ionospheric conditions in different days, a relative spatial change of $\triangle T E C$ on the order of some TECU [26]. A spectral shift of $200 \mathrm{kHz}$, corresponding to a baseline of $100 \mathrm{~m}$ and an incidence angle of $35^{\circ}$, is used. The relative spatial change of $\Delta \phi_{\Delta \text { iono }}$ is on the order of some cycles, whereas for $\Delta \phi_{\Sigma i o n o}$, it is on the order of some hundreds of cycle. A terrain slope of $30^{\circ}$ corresponds to a spectral shift of $1.7 \mathrm{MHz}$, which yields a phase difference between flat and oblique surfaces of one tenth of cycle. In this case, the second term of (35) can be then mostly ignored.

As second scenario, we consider a small acquisition in a low-latitude region; $\Sigma T E C=80$ TECU is constant within the image, and $\triangle \mathrm{TEC}=0$ TECU. The baseline is $1 \mathrm{~km}$, and the incidence angle is $35^{\circ}$. A terrain slope of $30^{\circ}$ generates a bias of almost two cycles with respect to flat zones. In this case, the final effect on ionosphere estimation after filtering should be considered, and countermeasures could be needed. 


\section{F. Asymmetric Split-Spectrum Mode}

Here, a special split-spectrum mode for SAR systems with larger bandwidth, such as the Tandem-L, ALOS-2, or NASAISRO SAR (NISAR) missions, is evaluated. This mode optimizes the compromise between data rate and accuracy. While wider bandwidths increase the accuracy of ionosphere estimation, the satellite onboard storage and downlink of a great amount of data is not always feasible. The optimum solution to still obtain a high accuracy with a smaller data volume would be to use two subbands of one third each of the allowed bandwidth, separated by one third. However, if the total used bandwidth is further reduced, to decrease the data amount, the ionosphere estimation noise grows. The new precision in estimating the ground movement using two subbands of bandwidth $B_{L}$ and $B_{H}$, from (18), is

$$
\sigma_{\Delta r_{\text {mov }}}^{\prime \prime}=\frac{c}{4 \pi f_{0}^{2}} \frac{f_{L} f_{H}}{f_{H}^{2}-f_{L}^{2}} \sqrt{\frac{f_{H}^{2}}{2 N_{L}}+\frac{f_{L}^{2}}{2 N_{H}}} \frac{\sqrt{1-\gamma^{2}}}{\gamma}
$$

where $N_{L}=N \cdot B_{L} / B$ and $N_{H}=N \cdot B_{H} / B$ are the numbers of independent samples of each subband. The ratio between this accuracy and the one obtained using the full bandwidth $B$ is

$$
\begin{aligned}
\frac{\sigma_{\Delta r_{\mathrm{mov}}}^{\prime \prime} \approx \frac{2 f_{0} B}{\sigma_{\Delta r_{\mathrm{mov}}}}}{9} & \sqrt{6 B \frac{B_{H}+B_{L}}{B_{H} B_{L}}} \frac{1}{f_{0}\left(2 B-B_{H}-B_{L}\right)} \\
& \cdot \frac{1}{B\left(B_{L}-B_{H}\right) / 2+\left(B_{H}^{2}-B_{L}^{2}\right) / 4} .
\end{aligned}
$$

For example, if we suppose to use one subband of $20 \mathrm{MHz}$ and one of $5 \mathrm{MHz}$, separated by the greatest possible distance inside the $85-\mathrm{MHz}$ allowed L-band spectrum, the accuracy of the splitspectrum method would be then 1.45 times worse than that obtained using an image with the full spectrum. This is anyway much better than only using $20 \mathrm{MHz}$; in that case, the accuracy would be eight times worse. In conclusion, even if the total used bandwidth is reduced due to data constraints, a small second subband, separated by the greatest possible distance inside the allowed spectrum, permits to obtain almost the same accuracy in estimating the ionosphere as when using the full spectrum.

This mode is tested in Section IV-D using high-resolution $85-\mathrm{MHz}$ ALOS-2 data to simulate a $20+5 \mathrm{MHz}$ acquisition.

In [27], it was shown that a custom chirp signal that concentrates all energy into the subbands increases the SNR with respect to a nominal chirp where a bandpass filter discards part of the energy to create the subbands. A special mode, which uses a modified chirp to increase the SNR, improving the accuracy (36), could be investigated in future work.

\section{Application Examples}

The split-spectrum method has been applied to four different ALOS PALSAR data sets to test its robustness and applicability. The first example is an interferogram of the 2008 Kyrgyzstan earthquake; it presents high coherence and smooth ionospheric variations. The excellent results show the correct separation between ground motion and ionospheric delay. The second
TABLE I

SCENES ACQUISITION INFORMATION

\begin{tabular}{cccccc}
\hline Example & Track(s) & Frame(s) & Master & Slave & N. \\
\hline Aurora & 243 & 1410 & $01 / 04 / 06$ & $17 / 05 / 06$ & 2 \\
\hline Kyrgyzstan & 527 & $750-790$ & $02 / 06 / 08$ & $20 / 08 / 09$ & 10 \\
\hline Wenchuan & 471 & $570-650$ & $29 / 02 / 08$ & $31 / 05 / 08$ & 18 \\
& 472 & $570-650$ & $28 / 01 / 07$ & $17 / 06 / 08$ & 18 \\
& 473 & $570-650$ & $17 / 02 / 08$ & $19 / 05 / 08$ & 18 \\
& 474 & $570-650$ & $05 / 03 / 08$ & $05 / 06 / 08$ & 18 \\
& 475 & $570-650$ & $20 / 06 / 07$ & $22 / 06 / 08$ & 18 \\
& 476 & $570-650$ & $08 / 04 / 08$ & $24 / 05 / 08$ & 18 \\
\hline ALOS-2 & 76 & 1280 & $10 / 09 / 14$ & $05 / 10 / 14$ & 2
\end{tabular}

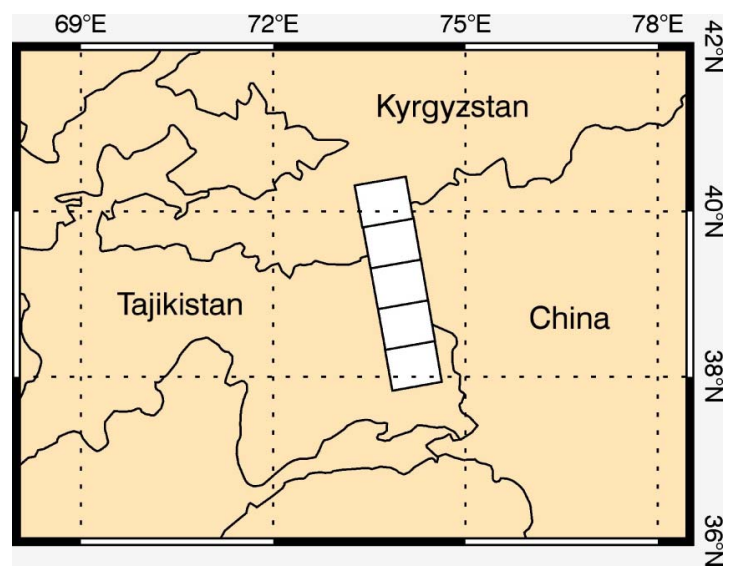

Fig. 4. Ground coverage of the PALSAR acquisitions for the Kyrgyzstan data set.

example is a measure of the aurora in northern Alaska. Due to the narrow 14-MHz bandwidth, the accuracy is low, and a big smoothing window is required. Consequently, small-scale variations of the ionosphere cannot be successfully recovered. A more challenging scenario, in the third example, is based on the 2008 Wenchuan earthquake. This data set is composed of 72 interferograms featuring low and high coherence levels. Phase unwrapping errors and low coherence are the main limitations; the separation between ionospheric and ground phases has been, however, achieved, and phase jumps between adjacent tracks have been reduced. All acquisitions were made during ascending passes; Table I reports detailed information about each scene.

\section{A. Kyrgyzstan 2008 Earthquake}

1) Data Set: On October 5, 2008, an earthquake struck the Nura region, in southern Kyrgyzstan [28]. We use SAR data to measure the coseismic surface displacements. The ALOS PALSAR images ground coverage is illustrated in the map in Fig. 4. With five pairs of ALOS PALSAR images, we generate an L-band interferogram, which is displayed in Fig. 5(a). The topographic phase was removed from the interferogram using a DEM. Apart from the earthquake, which is assumed to be localized only in the top part of the image, at least five fringes due to ionospheric variations can be seen in the bottom part of the interferogram. It is difficult to assess the real earthquake motion field since it is superimposed to the ionospheric phase screen. 


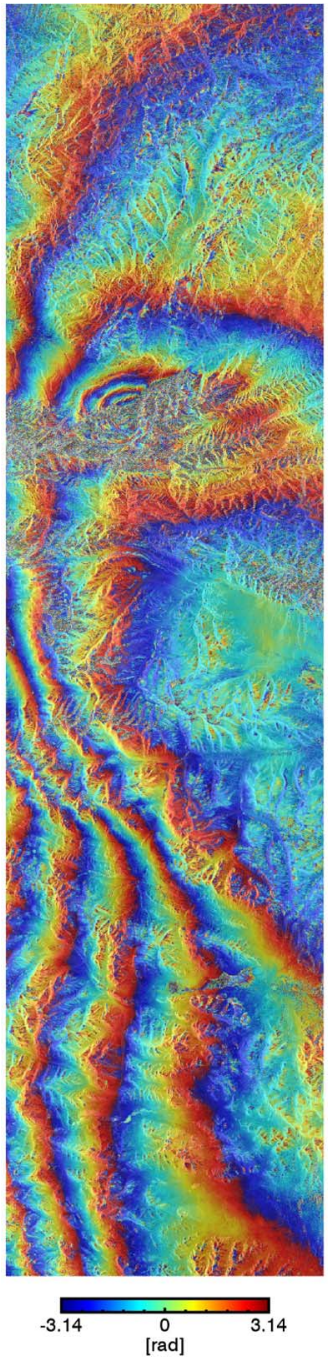

(a)

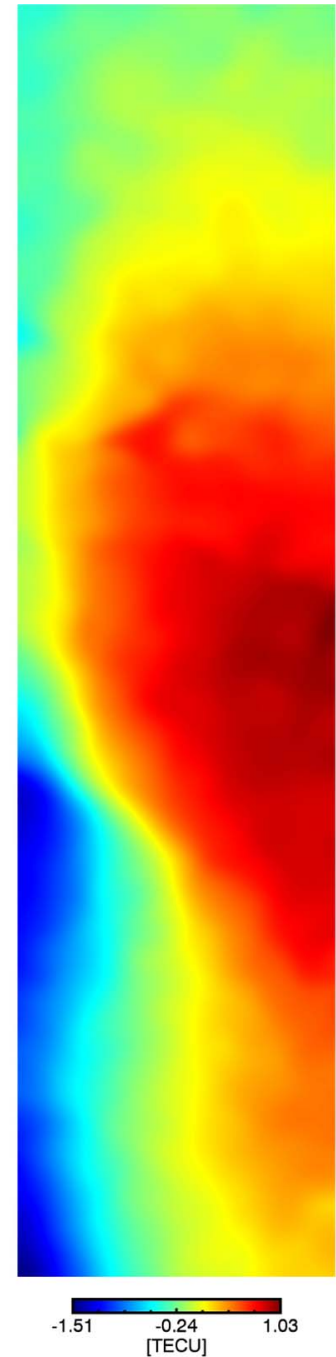

(b)

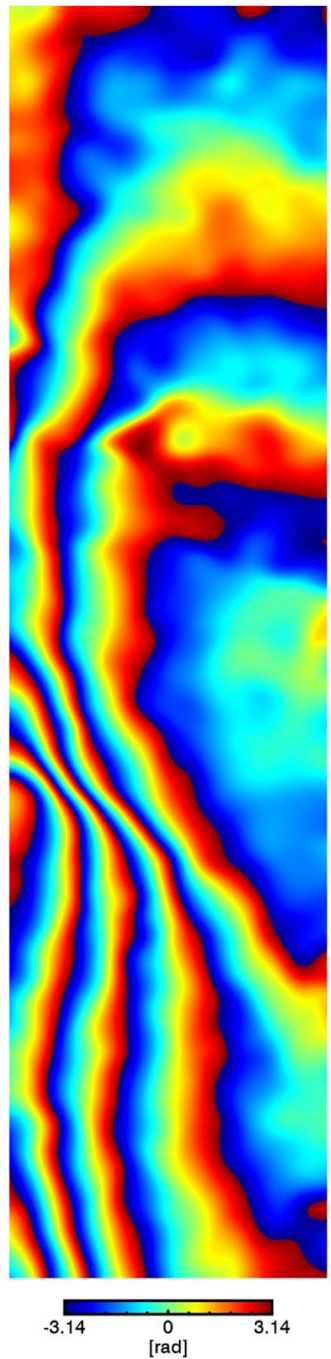

(c)

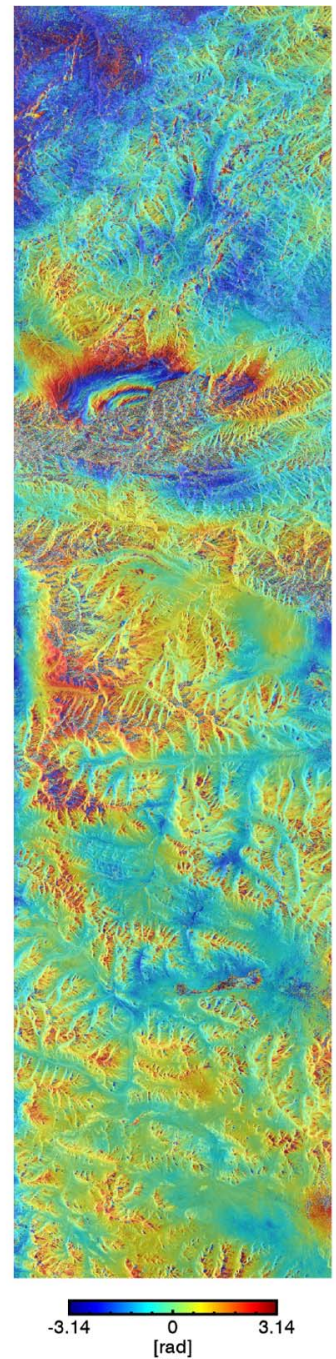

(d)

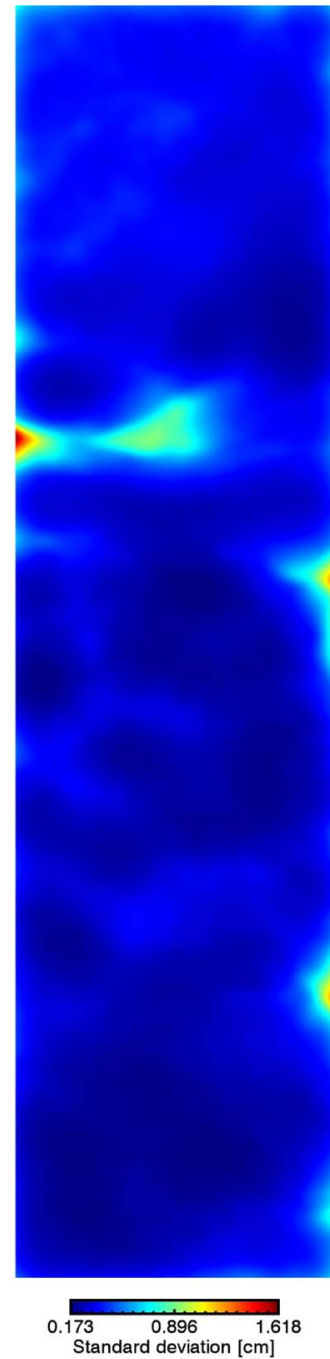

(e)

Fig. 5. (a) Kyrgyzstan 2008 earthquake of October 5 can be recognized in the top part of the interferogram. Five fringes in the bottom part of (a) are supposed to be due to ionosphere changes. (b) The ionospheric TEC map, estimated using the split-spectrum method, converted to a (c) phase screen, is used to produce the (d) ionosphere-compensated interferogram. (e) Expected accuracy of the ionosphere estimation. Azimuth length is $283 \mathrm{~km}$; range length is $68 \mathrm{~km}$.

The split-spectrum method was applied to the data set. Although the accuracy is limited by the narrow range bandwidth of $14 \mathrm{MHz}$, the mean coherence is 0.43 , and phase unwrapping was performed without problems. The ionosphere is relatively smooth; the point of fastest variation is in the middle of the image where five fringes are visible; this indicates a change of almost 3 TECU in about 45 ground $\mathrm{km}$. The images are oversampled by a factor 2 in both directions. The resulting oversampling is then 2.29 in range and 2.83 in azimuth. The applied multilooking is 23 pixels in range and 95 in azimuth; the resulting mean expected accuracy of the raw ionospheric estimates, which is calculated using (18), is $25 \mathrm{~cm}$. A Gaussian filtering with an $M$ parameter of 100 was used to increase the accuracy to about $2.5 \mathrm{~mm}$, as shown in Fig. 5(e). The output of the split-spectrum method [see Fig. 5(b)], converted to a phase screen [see Fig. 5(c)], is used to compensate the initial interferogram. The result [see Fig. 5(d)] shows how the ionospheric contribution was successfully removed. The earthquake pattern can be easily recognized in the top part of the image, whereas no motion is observed in the bottom part; the $60-\mathrm{cm}$ error that was introduced by five ionospheric fringes is now reduced to a millimeter level. Tropospheric delay is now more visible; in particular, a strong correlation of the phase with the topography indicates the presence of stratified tropospheric delay.

2) Performance Assessment: To check the performances of the method, the standard deviation of the raw ionosphere estimate has been calculated after the outlier rejection step. Results are shown in Fig. 6. The solid line represents the theoretical accuracy obtained from (18) considering the multilooking factor $23 \times 95$ and the oversampling. The asterisks and the circles represent the standard deviation of ionosphere estimates and are calculated in two different ways. For the asterisks, we used a moving window and the median absolute deviation, which is a robust estimator of the standard deviation. For the circles, we used the smooth ionosphere, which we suppose to be equal to the real ionospheric screen, to remove the mean value. We then take the squared error and average within different coherence intervals. The square root of the results is then displayed with their error bars. The good agreement between theoretical and measured accuracies confirm that the method performs as expected. 


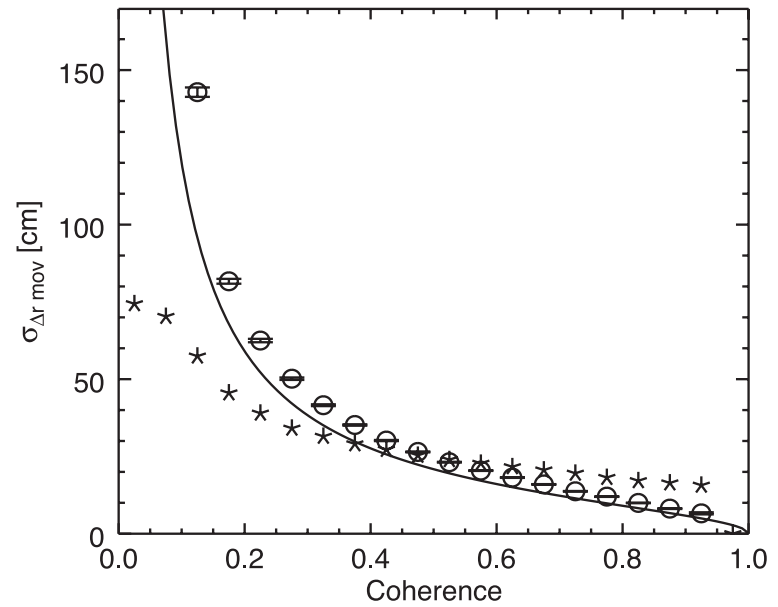

Fig. 6. Accuracy of the ionosphere estimate before filtering, Kyrgyzstan test case. The line is the theoretical accuracy, and the symbols are the measured standard deviation. Asterisks are calculated using a moving window and the median absolute deviation. Circles are calculated using the smooth ionosphere to remove the mean value and then the sample standard deviation.

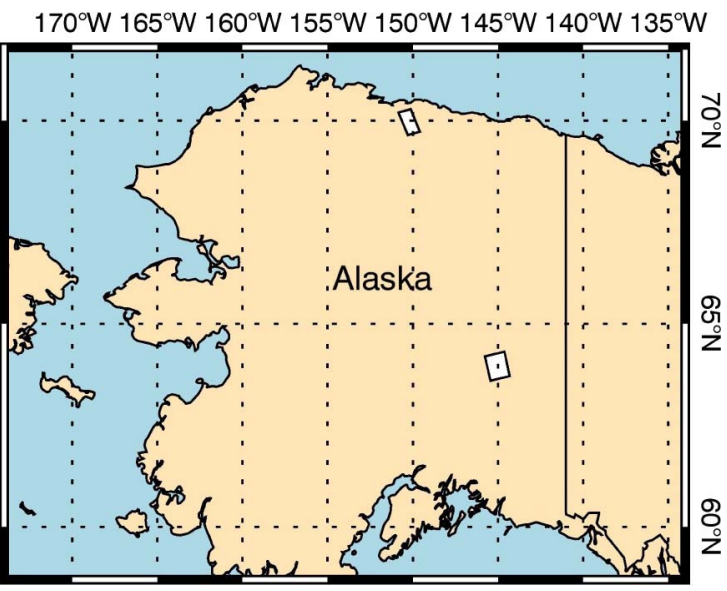

Fig. 7. In northern Alaska, the ground coverage of the PALSAR acquisition for the aurora data set; in eastern Alaska, the ALOS-2 acquisition.

\section{B. Aurora Borealis}

1) Data Set: The aurora borealis is caused by interactions between the solar wind and the Earth's atmosphere. Charged particles, which are carried by the solar wind and accelerated by the interconnections between the magnetic field of the Earth and that of the Sun, are conducted downward toward the magnetic poles where they collide with the atmosphere, ionizing oxygen and nitrogen atoms. A solar wind stream hit the Earth on March 31, 2006, causing visible auroras all around the north polar region for almost three days. L-band ALOS PALSAR images were acquired above Alaska during April 1, capturing the change of electron density in the ionosphere. The shape of the acquisition's ground coverage is represented in the map in Fig. 7. With a second acquisition in May 17, we produce an interferogram; the topographic phase was removed using a DEM.

The auroral activity generated strong and turbulent fluctuations in the ionospheric phase, as shown in the interferogram in Fig. 8. Coregistration is used to correct the azimuth shifts, which are caused by variations of the ionosphere along the flight path, recovering most of the coherence losses. In the left top corner, there is still a residual effect due to uncorrected

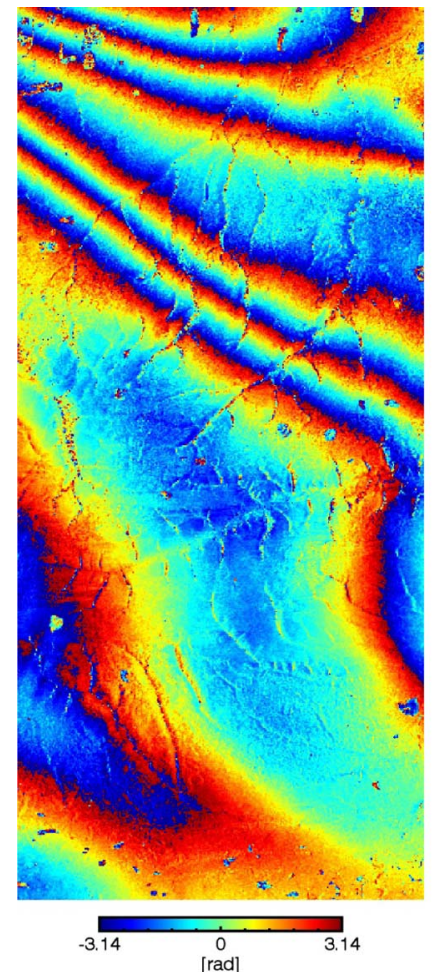

(a)

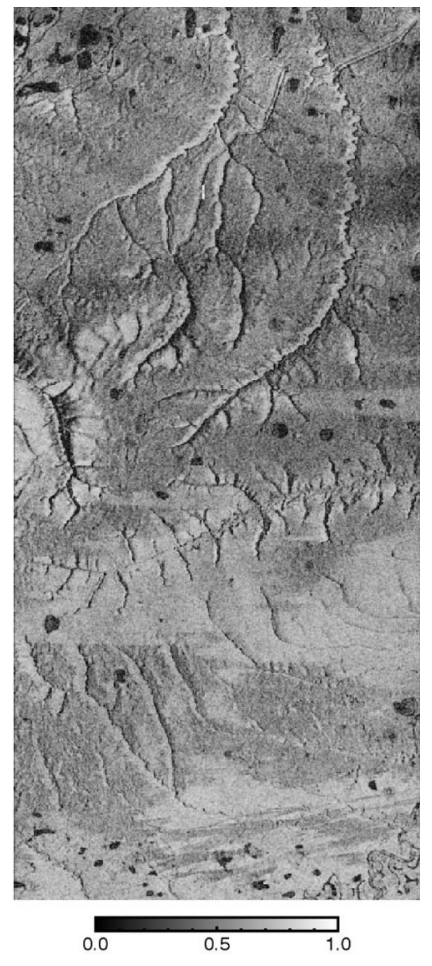

(b)
Fig. 8. Aurora activity generates rapid spatial variations in the ionospheric TEC; these variations are mapped in the (a) interferogram as phase changes. The (b) coherence shows almost no losses correlated with the ionosphere variations. Azimuth length is $66 \mathrm{~km}$; range length is $28 \mathrm{~km}$.

shift and/or blurring. The coherence features visible in the bottom half of the image are most probably related to changes in the ground backscatter. The along-track azimuth scale of these features is much smaller than the ionospheric azimuth resolution, which is some kilometers long. For this reason, we can exclude an ionospheric effect.

With a mean coherence of 0.5 , phase unwrapping is performed without problems. The size of this image is $66 \mathrm{~km}$ in azimuth and $28 \mathrm{~km}$ in range. In one half of the image, six fringes are visible; this means that the differential ionosphere varies of almost 3 TECU in about 33 ground $\mathrm{km}$. The total variation is similar to that of the previous example, but this time, it is less regular, and rapid undulations are present. Due to the relatively fine scale spatial variations of the phase screen, a small smoothing window would be preferred in order not to bias the output of the method. Unfortunately, the bandwidth is only $14 \mathrm{MHz}$, and the estimated phase is quite noisy.

2) Performance Assessment: To test this aspect, we calculate the root-mean-square deviation for increasing smoothing windows. The error is calculated between the output of the filter and the real ionospheric phase screen. Supposing no significant tropospheric contribution, the interferogram is used as real ionospheric phase screen. It is shown in Fig. 9 that the measured errors diverge from the theoretical ones when the filter size increases. The theoretical curve is the expected accuracy calculated with (18) considering the increasing effective number of looks $N_{\text {eff }}$. The measured error is composed of the estimation noise, which reduces for increasing filter sizes, and of the bias (the uncompensated high-frequency components), which, on the contrary, grows. 


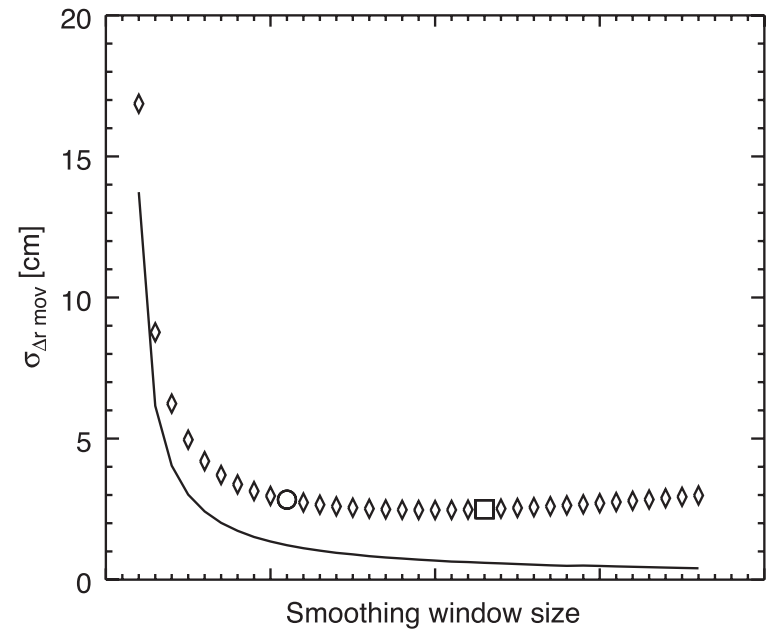

Fig. 9. Accuracy of filtered ionosphere estimate, aurora test case. Larger smoothing windows should theoretically give (solid line) lower errors; on the contrary, since small-scale ionospheric variations are not recovered, the (diamonds) measured error increases for larger smoothing windows.

Fig. 10 shows two examples of the filtered ionospheric phase screen and the respective compensated interferogram. Fig. 10(a) is produced using the filter size correspondent to the circle in Fig. 9. The result is very similar to the interferogram in Fig. 8, but a lot of irregularities, which can be also seen in the compensated interferogram in Fig. 10(b), are still present. They derive from the estimation noise, being unlikely due to atmospheric delay. A larger filtering window, correspondent to the square in Fig. 9, is used to produce Fig. 10(c). This ensures a smoother result, but the compensated interferogram still show residuals, which are the cause of the higher error, with respect to the expected, in Fig. 9. The residuals can be attributed to a bias between the real and estimated ionospheric phase screens. There is a tradeoff between the increase of the accuracy obtained with more smoothing and the biases that too much smoothing can originate. An adaptive filter, which should reduce the noise variance but also respect the highfrequency components of the phase screen, could be used to improve the result. Another possibility, to make the phase screen more precise, is the combination of more ionosphere estimation methods. Anyway, despite this issue, the method was able to reduce the error from approximately $60 \mathrm{~cm}$ to some centimeters.

\section{Wenchuan 2008 Earthquake}

1) Data Set: On May 12, 2008, an earthquake struck the Wenchuan region in central China. The set of images shown in Fig. 11 is what is typically selected by researchers for studying coseismic deformation patterns [29].

This is because the acquisition dates of these images reduce the influence of postseismic deformation on the interferograms. Unfortunately, this image set is heavily influenced by ionospheric distortions and needs to be corrected to enable thorough geophysical modeling. The ionospheric disturbances are superimposed on the ground motion signal and are clearly visible in the set of interferograms in Fig. 12(a). To cover the whole earthquake, many adjacent tracks have to be joined. Since each track was acquired on a different day, each one experienced

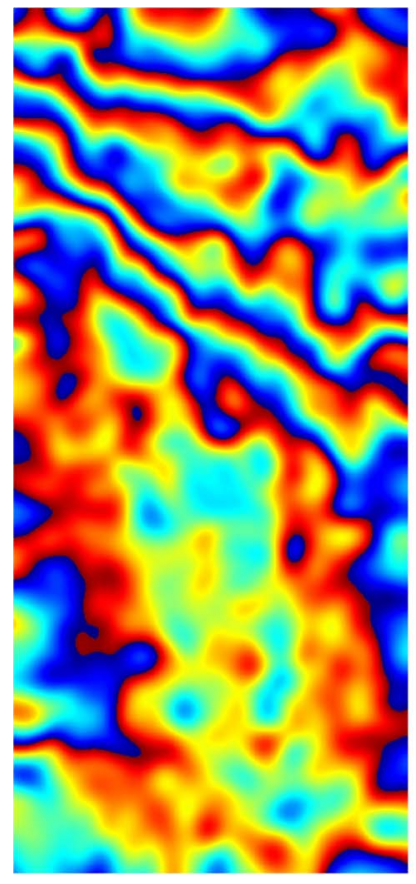

(a)

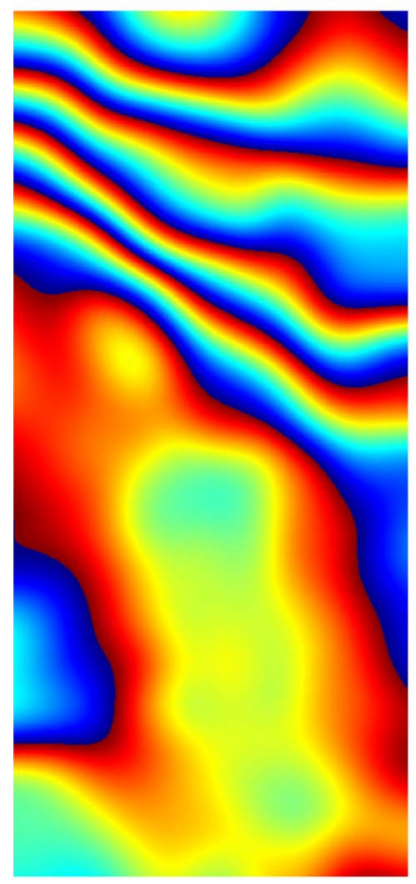

(c)

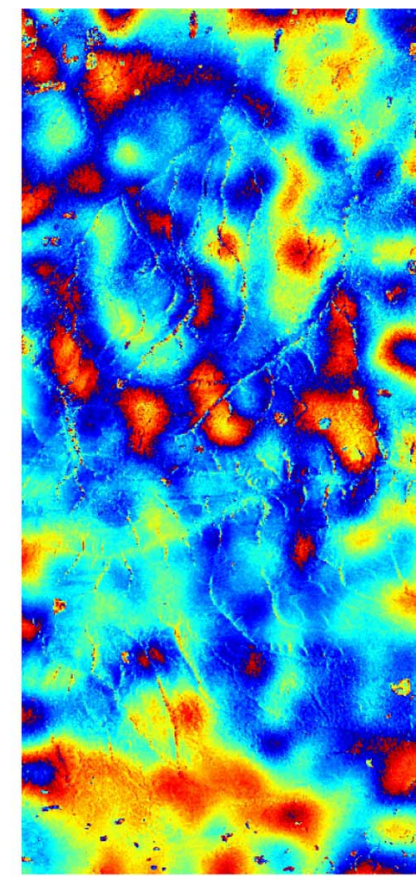

(b)

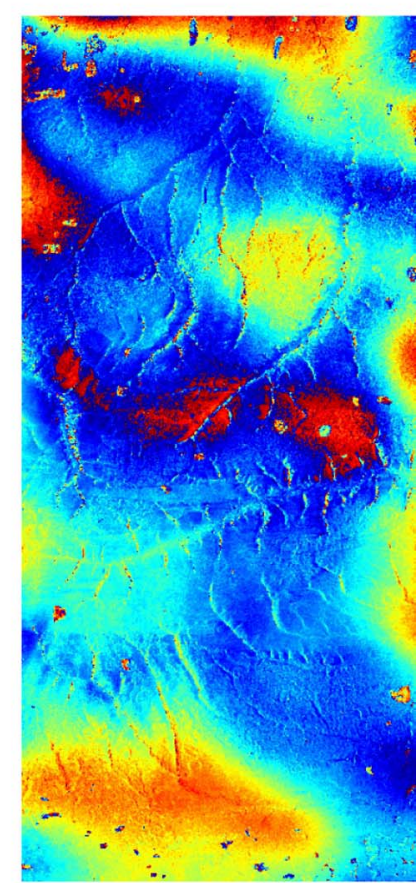

(d)
Fig. 10. (a) and (c) Estimated ionospheric phase screens. (b) and (d) Compensated interferograms. The larger smoothing window in (c), with respect to (a), ensures smoother but biased results. The color bar goes from $-\pi$ to $\pi$ for all images.

a different ionosphere. A discrepancy between adjacent tracks can be expected due to aftershocks motion, different looking angles, and tropospheric delay. However, the strong phase jumps present in the interferogram in Fig. 12(a), particularly far away from the earthquake, are an indication of ionospheric activity. Moreover, strong residuals between the range deformation predicted by the geophysical model and the InSAR deformation [29] are a further indication of the ionosphere presence. 


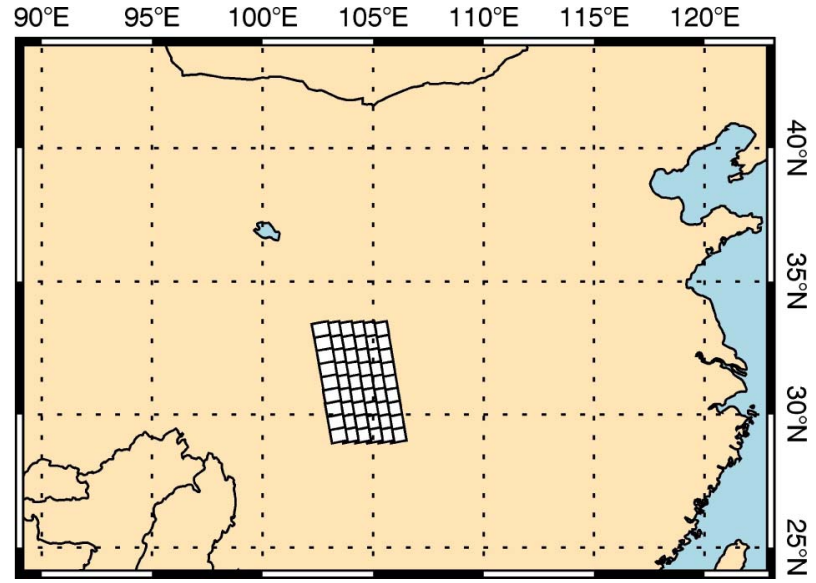

Fig. 11. Ground coverage of the PALSAR acquisitions for the Wenchuan data set. The azimuth length of nine consequent images is $510 \mathrm{~km}$; the range length of six adjacent tracks is $480 \mathrm{~km}$.

This data set is composed of 72 interferograms, and the coherence spans from low to high. The bandwidth is $28 \mathrm{MHz}$ for all tracks, except one (475). The differential phase unwrapping errors in the areas of low coherence were corrected as presented in Section III-C. An example of this correction is presented in the next paragraph. The split-spectrum method output is subtracted from the interferogram, and the result is shown in Fig. 12(b). Ionospheric-induced errors in the ground motion estimation, of about 50 centimeters, have been thus removed. The size of the filtering window has been adapted to the mean coherence of each track to obtain an almost homogeneous accuracy of about $3 \mathrm{~mm}$. It can be seen how the motion is now only localized around the fault and how phase jumps between different tracks are greatly reduced. Remaining discrepancies can be attributed to tropospheric delay and aftershock motion. The linear trend in the first track from the right could be caused by an orbit error.

2) Phase Unwrapping Errors Correction: Here, we show the effects of the unwrapping errors correction. In Fig. 13(a), the original interferogram used for this example is displayed. It is one frame of the first track from the left, just beneath the earthquake rupture. Fig. 13(d) and (e) shows the raw ionospheric phase estimates before and after the correction. Biases, which are due to differential phase unwrapping error, are present in the uncorrected estimates and are successfully eliminated in the corrected one. Fig. 13(f) and (g) shows the relative filtered phase screens, whereas Fig. 13(b) and (c) shows the ionosphere-compensated interferograms.

\section{Asymmetric Split-Spectrum Mode}

To validate the theoretical performance for $20+5 \mathrm{MHz}$ SAR acquisitions that was developed in Section III-F, here, we analyze two 85-MHz ALOS-2 acquisitions over Alaska. The ground coverage is displayed in Fig. 7; the size of the images is $57 \mathrm{~km}$ range and $69 \mathrm{~km}$ azimuth. This mode is intended to reduce the amount of data but still preserve the ionosphere estimation accuracy. Two subbands of 20 and $5 \mathrm{MHz}$ are produced, at the two ends of the full available spectrum, with bandpass filtering.

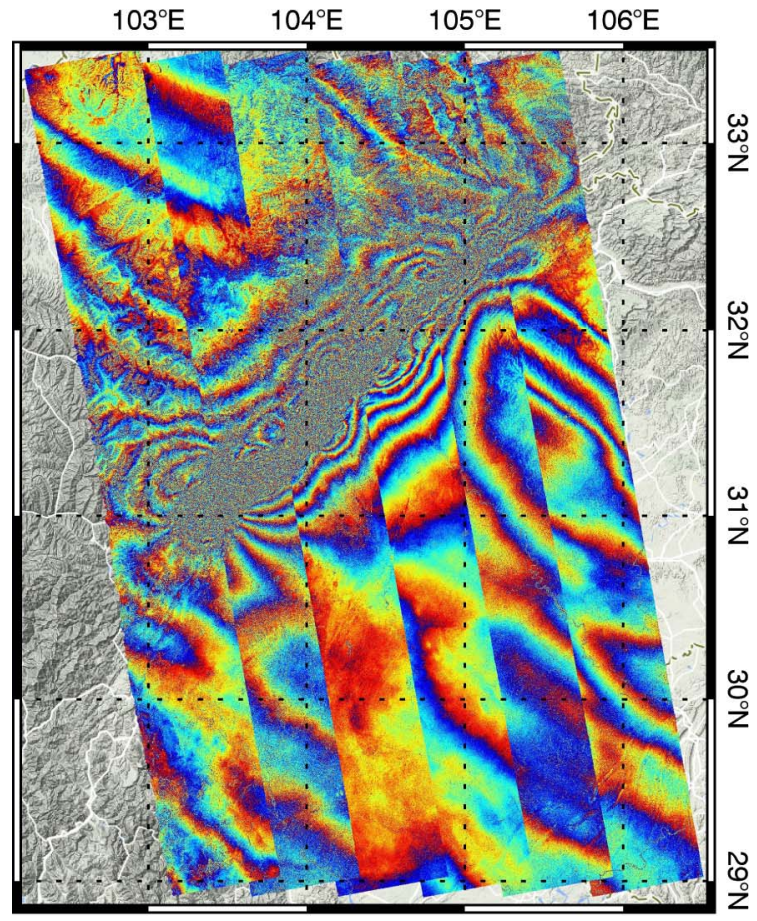

(a)

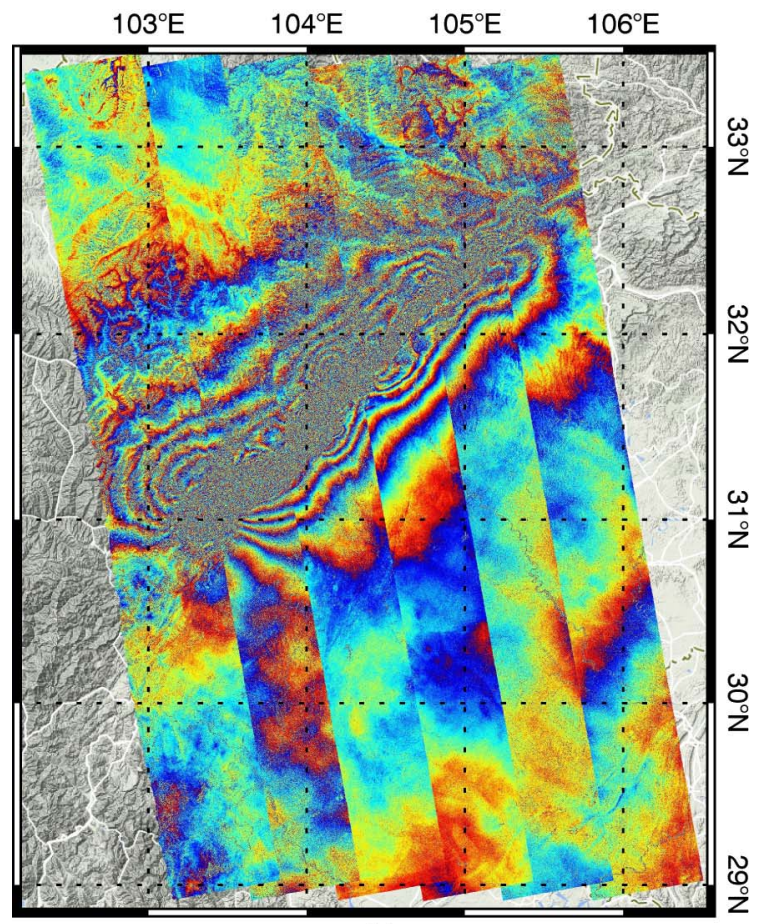

(b)

Fig. 12. (a) Original 2008 Wenchuan Earthquake interferogram. (b) After ionosphere compensation.

The split-spectrum method is then applied to the $85-\mathrm{MHz}$ and $20+5 \mathrm{MHz}$ acquisitions. The theoretical and measured standard deviations of the raw ionospheric phase estimate for both implementations are reported in Fig. 14. The curves of the expected accuracy are calculated from (18) and (36), considering the multilooking factor $11 \times 16$. The ionosphere estimation accuracy obtained using asymmetrical subbands is close to that obtained with the complete bandwidth. The advantage of using 


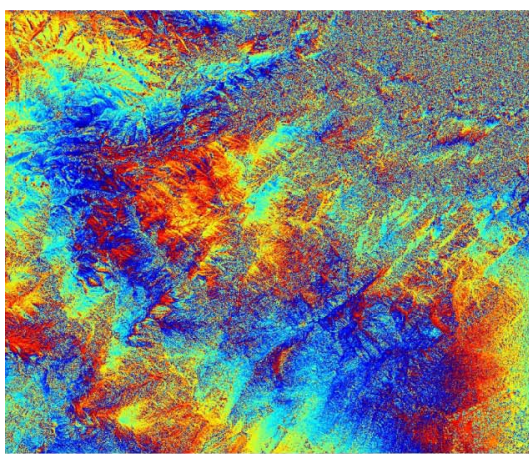

(a)

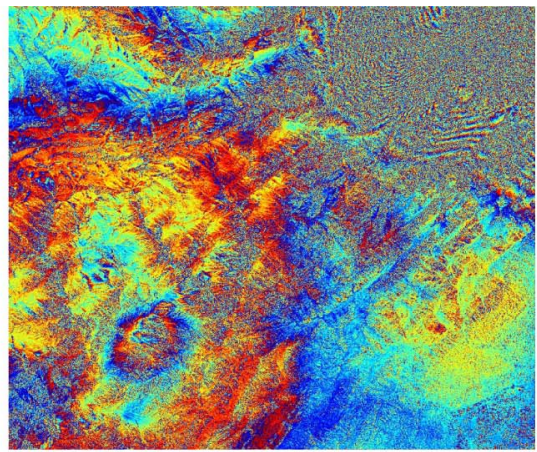

(b)

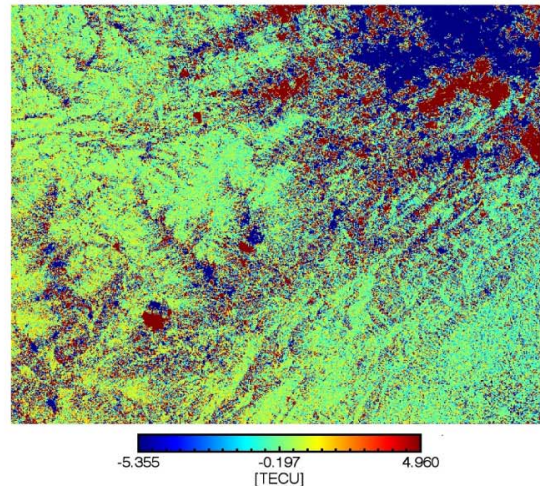

(d)

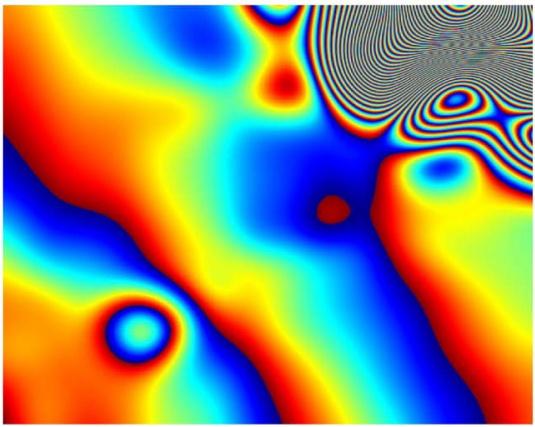

(f)

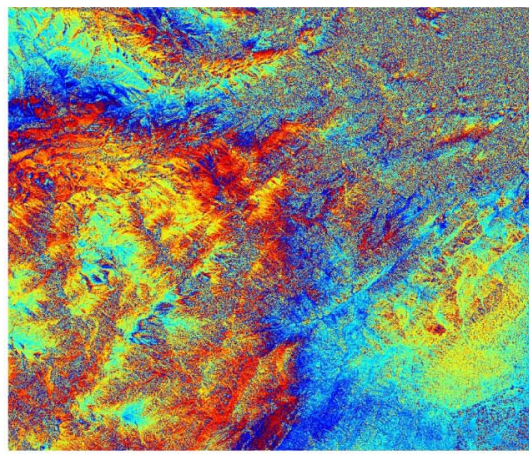

(c)

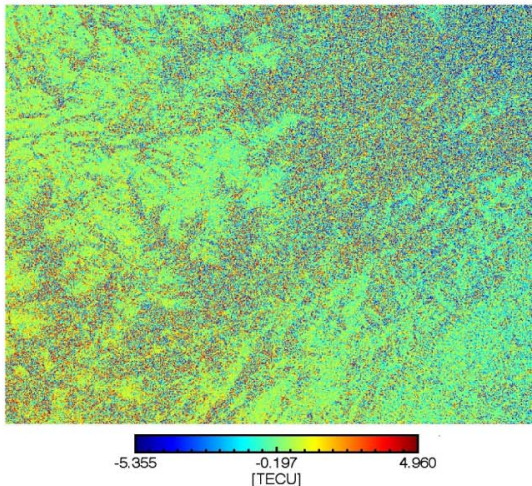

(e)

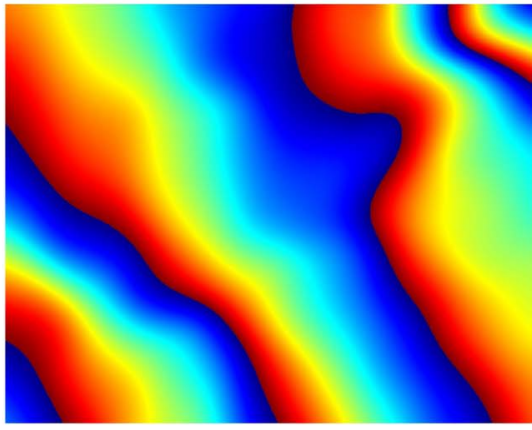

(g)

Fig. 13. Effect of the phase unwrapping errors correction. (a) Original interferogram. (b) and (c) Corrected interferograms without and with correction. (d) and (e) Raw ionosphere estimates without and with correction. (f) and (g) Respective filtered phase screens.

a small second subband at the other end of the available spectrum is demonstrated; it allows a reduction of the bandwidth to save downlink and memory capacity without sacrificing the compensation of ionospheric disturbances. The good agreement between expected and measured accuracies proves again the precision of the assumptions.

The full bandwidth interferogram and the compensated one are presented in Fig. 15.

\section{CONCLUSION}

The split-spectrum method is an important element toward the realization of an operational processor for compensation of ionospheric effects in SAR interferograms. In this paper, we presented and tested an implementation of the method, which estimates the ionospheric phase. The final estimation accuracy depends on the carrier frequency and bandwidth of the images, on the interferometric coherence, and on the correlation length of the differential ionosphere. For example, using typical L-band images with $28-\mathrm{MHz}$ bandwidth and coherence 0.6 , it is necessary to average over a ground area of about $1 \mathrm{~km}^{2}$ to reach the accuracy of $1 \mathrm{~cm}$. To increase the accuracy up to $1 \mathrm{~mm}$, it is necessary to use a ground area of about $100 \mathrm{~km}^{2}$.

We applied the method to four data sets of ALOS and ALOS-2 images, each with different characteristics. The Kyrgyzstan earthquake example (see Section IV-A) shows the correct separation between the ground movements and troposphere and the ionospheric phase. Despite the narrow 14-MHz bandwidth, it was possible to reach millimeter accuracy. The aurora borealis example (see Section IV-B), on the other hand, shows how small-scale ionospheric variations limit the amount of allowable filtering and, hence, the estimation and correction performance that can be achieved by the algorithm. The Wenchuan earthquake example (see Section IV-C) demonstrates the 


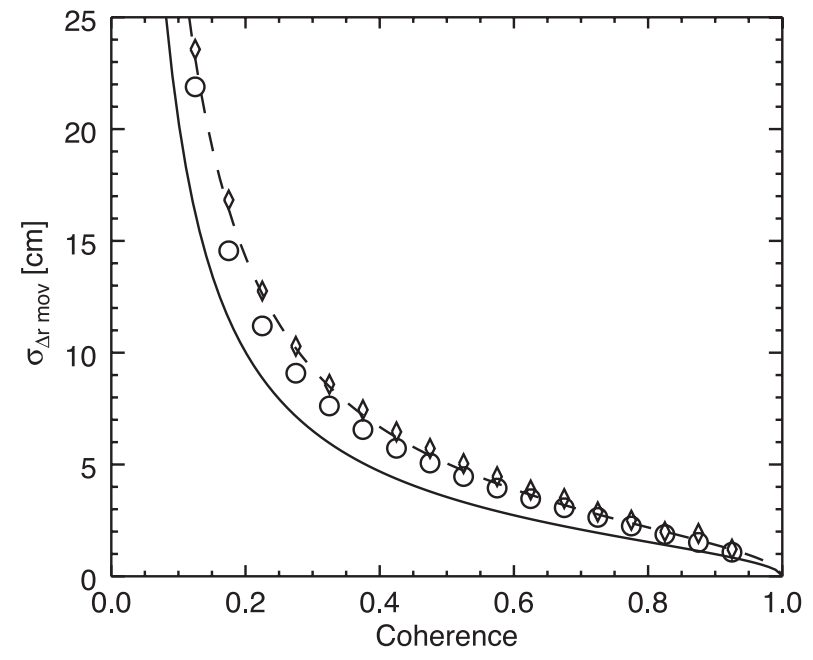

Fig. 14. Expected accuracy of the $85-\mathrm{MHz}$ acquisition is represented by the black line, whereas the circles are the measured standard deviation. The dashed line is the expected accuracy for the $20+5 \mathrm{MHz}$ acquisition; diamonds are the measured standard deviation.

robustness of the method, being applied to 72 interferograms with different characteristics. In these examples, an ionospheric error of almost $1 \mathrm{~m}$ has been reduced to millimeter or centimeter level. The asymmetric split-spectrum mode, tested using ALOS-2 images in Section IV-D, shows how it is possible to reduce the bandwidth and thus the data amount but still estimate the ionospheric phase with almost the same accuracy as when using full-band images.

It is conceivable to extend this work in various directions; possible future works are discussed in the following paragraphs. The combination of precise differential ionosphere variations, which are obtained from SAR images, and absolute measurements can lead to the development of an ionospheric mapping system with high spatial resolution and accuracy. Absolute TEC values can be obtained from GNSS measurements or from the SAR images, e.g., by exploiting the quadratic behavior of the ionospheric phase or the wavenumber shift effect reported in Section III-E. These methods have been already proposed, but they still have to be developed and demonstrated.

SAR systems working with different frequencies other than L-band can benefit from the split-spectrum correction method, too. Spatial phase undulations generated by ionospheric variations are often attributed to troposphere or orbit errors and removed with polynomial fitting of uncertain accuracy. The split-spectrum processing can be now used to precisely correct the ionospheric contribution. More testing is required to prove the importance of ionospheric effects in C-band or X-band images.

An extension of the split-spectrum method to point scatterers and stacks of images is a topic worthy of further investigations.

Regarding the improvement of the processor, we know that ionospheric variations with smaller spatial scales than the filtering window are not recovered and bias the result, lowering the accuracy of the final estimate. Adaptive filtering of the raw ionosphere estimate can improve the final phase screen. Alternatively, we propose to use the azimuth shifts, which are estimated by cross correlation or spectral diversity, to increase

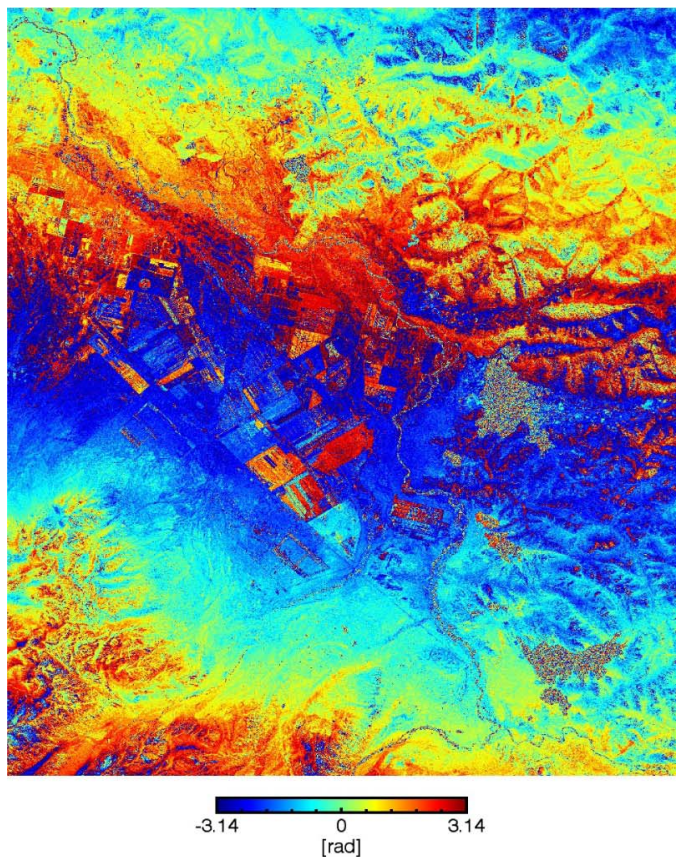

(a)

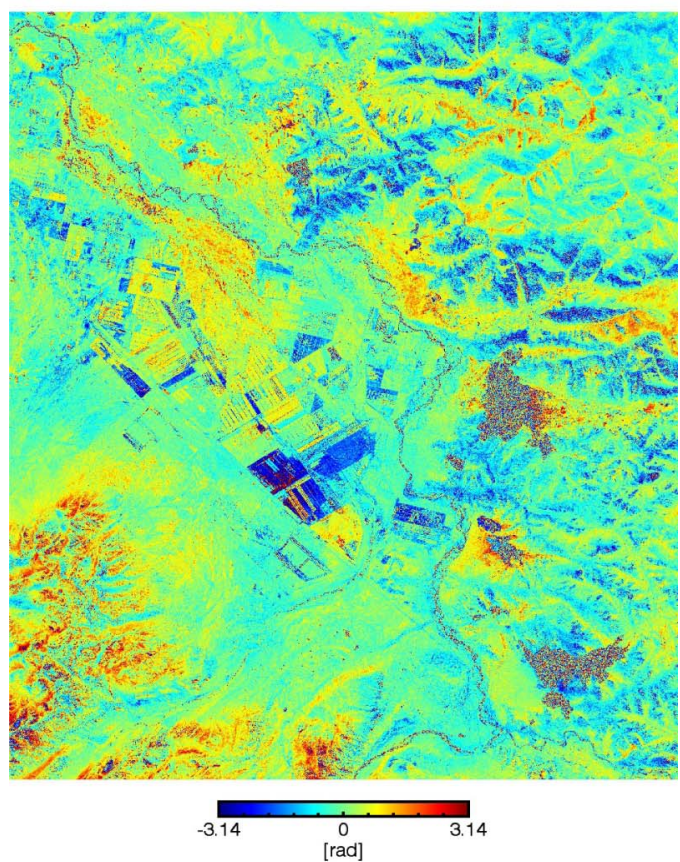

(b)

Fig. 15. ALOS-2 85-MHz interferogram over Alaska. (a) Original. (b) Ionosphere-compensated version. Azimuth length is $69 \mathrm{~km}$; range length is $57 \mathrm{~km}$.

the accuracy of the ionospheric phase estimate. Subapertures will be used to separate the ionosphere-induced azimuth shifts from the ground movements. Being sensitive to local azimuth variations of the ionosphere, the azimuth shifts can estimate the high-frequency components of the ionosphere spectrum but are prone to an increasing error in the long distance and are insensitive to range variations. The split-spectrum method ensures accurate estimation over long wavelengths and can recover range variations. The two techniques will therefore complement each other in the realization of an operational processor. 


\section{ACKNOWLEDGMENT}

The authors would like to thank Dr. R. Brcic for his previous work on the split-spectrum method, the anonymous reviewers who helped to improve the quality of this paper, and the Japanese Aerospace Exploration Agency for providing the PALSAR data for this study (in part under the ALOS RA-4 proposal PI1118). The authors also acknowledge the support of the "Helmholtz Alliance Remote Sensing and Earth System Dynamics."

\section{REFERENCES}

[1] Z.-W. Xu, J. Wu, and Z.-S. Wu, "A survey of ionospheric effects on space-based radar," Waves Random Media, vol. 14, no. 2, pp. S189-S273, Apr. 2004.

[2] F. Meyer, "Performance requirements for ionospheric correction of lowfrequency SAR data," IEEE Trans. Geosci. Remote Sens., vol. 49, no. 10, pp. 3694-3702, Oct. 2011.

[3] N. Jakowski, C. Mayer, M. M. Hoque, and V. Wilken, "Total electron content models and their use in ionosphere monitoring," Radio Sci., vol. 46, no. 6, 2011, Art ID. RS0D18.

[4] F. Meyer, "A review of ionospheric effects in low-frequency SARSignals, correction methods, and performance requirements," in Proc. IEEE IGARSS, Jul. 2010, pp. 29-32.

[5] R. Brcic, A. Parizzi, M. Eineder, R. Bamler, and F. Meyer, "Estimation and compensation of ionospheric delay for SAR interferometry," in Proc. IEEE IGARSS, 2010, pp. 2908-2911.

[6] P. Rosen, S. Hensley, and C. Chen, "Measurement and mitigation of the ionosphere in L-band interferometric SAR data," in Proc. IEEE Radar Conf., May 2010, pp. 1459-1463.

[7] F. Meyer, R. Bamler, N. Jakowski, and T. Fritz, "The potential of low-frequency SAR systems for mapping ionospheric TEC distributions," IEEE Geosci. Remote Sens. Lett., vol. 3, no. 4, pp. 560-564, Oct. 2006.

[8] D. Raucoules and M. de Michele, "Assessing ionospheric influence on L-band SAR data: Implications on coseismic displacement measurements of the 2008 Sichuan earthquake," IEEE Geosci. Remote Sens. Lett., vol. 7, no. 2, pp. 286-290, Apr. 2010.

[9] J. S. Kim, A. Danklmayer, and K. Papathanassiou, "Correction of ionospheric distortions in low frequency interferometric SAR data," in Proc. IEEE IGARSS, Jul. 2011, pp. 1505-1508.

[10] H.-S. Jung, D.-T. Lee, Z. Lu, and J.-S. Won, "Ionospheric correction of SAR interferograms by multiple-aperture interferometry," IEEE Trans. Geosci. Remote Sens., vol. 51, no. 5, pp. 3191-3199, May 2013.

[11] A. Freeman and S. Saatchi, "On the detection of Faraday rotation in linearly polarized L-band SAR backscatter signatures," IEEE Trans. Geosci. Remote Sens., vol. 42, no. 8, pp. 1607-1616, Aug. 2004.

[12] A. Freeman, "Calibration of linearly polarized polarimetric SAR data subject to Faraday rotation," IEEE Trans. Geosci. Remote Sens., vol. 42, no. 8, pp. 1617-1624, Aug. 2004.

[13] F. Meyer and J. Nicoll, "Prediction, detection, and correction of Faraday rotation in full-polarimetric L-band SAR data," IEEE Trans. Geosci. Remote Sens., vol. 46, no. 10, pp. 3076-3086, Oct. 2008.

[14] D. Belcher, "Theoretical limits on SAR imposed by the ionosphere," Radar, Sonar Navigat., IET, vol. 2, no. 6, pp. 435-448, Dec. 2008.

[15] R. Hanssen, Radar Interferometry: Data Interpretation and Error Analysis. Boston, MA, USA: Kluwer, 2001.

[16] S. Tebaldini, A. M. Guarnieri, and F. Rocca, "Recovering time and space varying phase screens through SAR multi-squint differential interferometry," in Proc. 9th EUSAR, Apr. 2012, pp. 16-19.

[17] G. Gomba, M. Eineder, A. Parizzi, and R. Bamler, "High-resolution estimation of ionospheric phase screens through semi-focusing processing," in Proc. IEEE IGARSS, Jul. 2014, pp. 17-20.

[18] S. Kay, Fundamentals of Statistical Signal Processing: Estimation Theory, ser. Fundamentals of Statistical Signal Processing. Englewood Cliffs, NJ, USA: Prentice-Hall, 1993.

[19] F. De Zan, "Coherent shift estimation for stacks of SAR images," IEEE Geosci. Remote Sens. Lett., vol. 8, no. 6, pp. 1095-1099, Nov. 2011.

[20] B. Friedlander, "On the Cramer-Rao bound for time delay and Doppler estimation," IEEE Trans. Inf. Theory, vol. IT-30, no. 3, pp. 575-580, May 1984
[21] R. Bamler and M. Eineder, "Accuracy of differential shift estimation by correlation and split-bandwidth interferometry for wideband and delta-k SAR systems," IEEE Geosci. Remote Sens. Lett., vol. 2, no. 2, pp. 151155, Apr. 2005.

[22] M. Seymour and I. Cumming, "Maximum likelihood estimation for SAR interferometry," in Proc. IGARSS, Surface and Atmospheric Remote Sensing: Technologies, Data Analysis and Interpretation, 1994, vol. 4, pp. 2272-2275

[23] F. Gatelli et al., "The wavenumber shift in SAR interferometry," IEEE Trans. Geosci. Remote Sens., vol. 32, no. 4, pp. 855-865, Jul. 1994.

[24] F. De Zan, P. Prats-Iraola, and M. Rodriguez-Cassola, "On the dependence of delta-k efficiency on multilooking," IEEE Geosci. Remote Sens. Lett., vol. 12, no. 8, pp. 1745-1749, Aug. 2015.

[25] G. Krieger et al., "Tropospheric and ionospheric effects in spaceborne single-pass SAR interferometry and radargrammetry," in Proc. 10th EUSAR, Jun. 2014, pp. 1-4.

[26] K. Davies, Ionospheric Radio. Stevenage, U.K.: IET, 1990, no. 31.

[27] R. Brcic, M. Eineder, and R. Bamler, "Interferometric absolute phase determination with TerraSAR-X wideband SAR data," in Proc. IEEE Radar Conf., May 2009, pp. 1-6.

[28] K. Teshebaeva, H. Sudhaus, H. Echtler, B. Schurr, and S. Roessner, "Strain partitioning at the eastern Pamir-Alai revealed through SAR data analysis of the 2008 Nura earthquake," Geophys. J. Int., vol. 198, no. 2, pp. 760-774, Jun. 2014.

[29] Z.-K. Shen et al., "Slip maxima at fault junctions and rupturing of barriers during the 2008 Wenchuan earthquake," Nature Geosci., vol. 2, no. 10 , pp. $718-724,2009$.

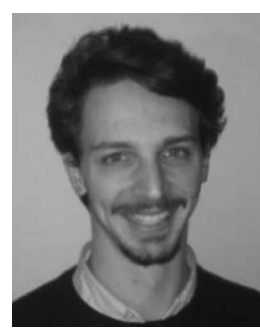

Giorgio Gomba received the Bachelor's and Master's degrees in telecommunication engineering from Politecnico di Milano, Milano, Italy, in 2009 and 2011, respectively. He is currently working toward the Ph.D. degree at the Technical University of Munich (TUM), Munich, Germany.

Since 2012, he has been with the Remote Sensing Technology Institute, German Aerospace Center (DLR), Wessling, Germany. His current research interests are the estimation and compensation of ionospheric propagation delay in synthetic aperture

radar signals

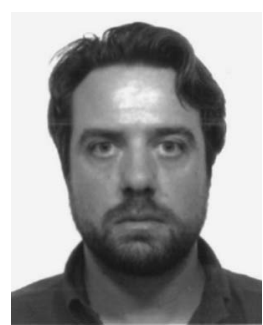

Alessandro Parizzi received the Master's degree in telecommunications engineering from Politecnico di Milano, Milano, Italy, in 2004.

From 2004 to 2006, he was with the Department of Electronics, Politecnico di Milano, working in the field of SAR interferometry. Since 2006, he has been with the Remote Sensing Technology Institute, German Aerospace Center (DLR), Wessling, Germany, working on interferometric SAR processing. His main research interest is related to the study of atmospheric and decorrelation disturbances in differential SAR interferometry.

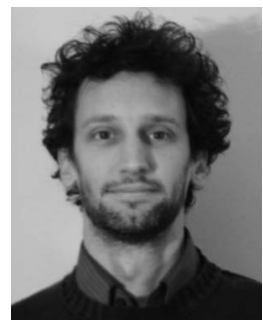

Francesco De Zan received the Master's degree in telecommunication engineering and the Ph.D. degree from Politecnico di Milano, Milano, Italy, in 2004 and 2008, respectively.

During his Ph.D. studies, he worked on extending permanent scatter interferometry to decorrelating targets and contributed to European Space Agency studies in preparation for Sentinel-1. Since 2008, he has been with the German Aerospace Center (DLR), Wessling, Germany, initially at the Microwaves and Radar Institute and then at the Remote Sensing Technology Institute. He has been involved in studies for numerous future interferometric SAR missions and in calibration activities of the TanDEM-X interferometer. His research interests include algorithms and theoretical bounds for shift estimation and SAR interferometry and interpretation of phase and coherence signatures. 


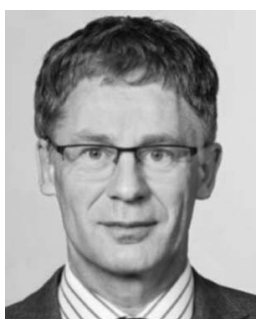

Michael Eineder (SM'01) received the Diploma degree in electrical engineering from Technische Universität München (TUM), München, Germany, in 1990 and the Dr. rer. nat. degree from the University of Innsbruck, Innsbruck, Austria, in 2004.

Since 2013, he has been an honorary Professor with TUM, where he is also currently a part-time Lecturer of remote sensing. He is also currently the Head of the SAR signal processing department of the Remote Sensing Technology Institute, German Aerospace Center (DLR), Wessling, Germany. His responsibilities encompass the development of SAR and interferometric SAR processing systems for current and future radar missions. Since 1990, he has been with DLR, where he has worked on a variety of international missions, including SIR-C/X-SAR, SRTM/X-SAR in cooperation with the National Aeronautics and Space Administration, ERS-1 (European Space Agency), and TerraSAR-X and TanDEM-X (Germany). His current research interest is focused on future SAR missions and on imaging geodesy, an absolute positioning technique exploiting high-resolution SAR.

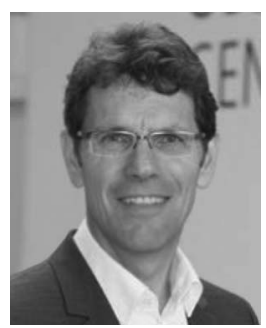

Richard Bamler (M'95-SM'00-F'05) received the Diploma degree in electrical engineering, the Doctorate degree in engineering, and the Habilitation degree in the field of signal and systems theory from Technische Universität München (TUM), München, Germany, in 1980, 1986, and 1988, respectively.

From 1981 to 1989 , he worked on optical signal processing, holography, wave propagation, and tomography at the university. In 1989, he joined the German Aerospace Center (DLR), Wessling, Germany, where he is currently the Director of the Remote Sensing Technology Institute. In early 1994, he was a Visiting Scientist with the Jet Propulsion Laboratory in preparation of the SIC-C/X-SAR missions. In 1996, he was a Guest Professor with the University of Innsbruck, Innsbruck, Austria. Since 2003, he has also held a full professorship in remote sensing technology at TUM as a double appointment with his DLR position. His teaching activities include university lectures and courses on signal processing, estimation theory, and SAR. He, his team, and his institute have been working on SAR and optical remote sensing, image analysis and understanding, stereo reconstruction, computer vision, ocean color, passive and active atmospheric sounding, and laboratory spectrometry. They were and are responsible for the development of the operational processors for SIR-C/X-SAR, SRTM, TerraSAR-X, TanDEM-X, Tandem-L, ERS-2/GOME, ENVISAT/SCIAMACHY, MetOp/GOME-2, Sentinel 5p, EnMAP, etc. His research interests are in algorithms for optimum information extraction from remote sensing data with emphasis on SAR. This involves new estimation algorithms, such as sparse reconstruction and compressive sensing. He has devised several high-precision algorithms for monostatic and bistatic SAR processing, SAR calibration and product validation, Ground Moving Target Indicator (GMTI) for traffic monitoring, SAR interferometry, phase unwrapping, persistent scatterer interferometry, and differential SAR tomography and data fusion. 